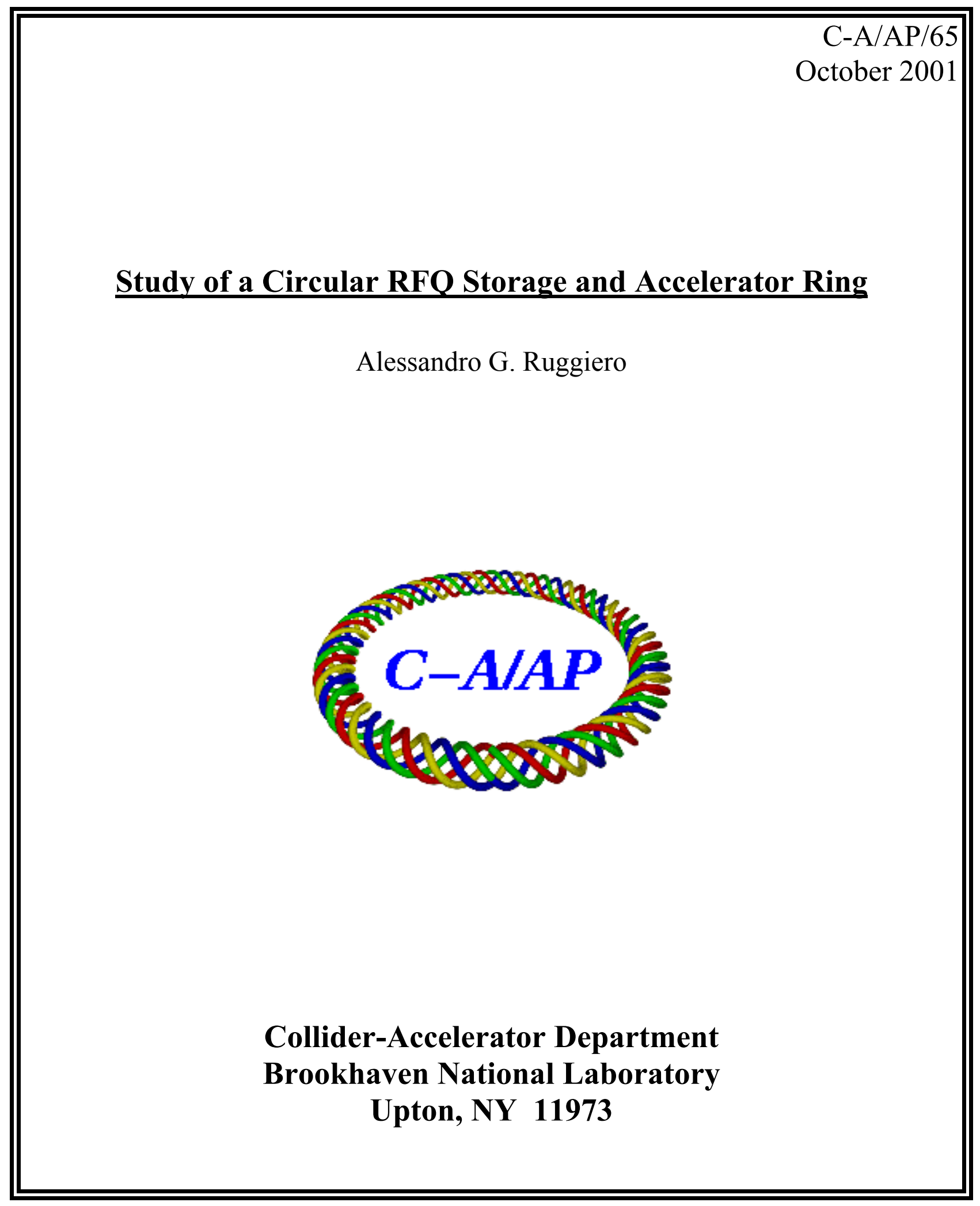




\title{
Study of a Circular RFQ Storage and Accelerator Ring*
}

\author{
Alessandro G. Ruggiero \\ Brookhaven National Laboratory \\ October 2001
}

\section{Abstract}

The Circular Radio-Frequency Quadrupole (CRFQ) is a new concept of storage and accelerator ring for intense beams of light and heavy ions, protons and electrons. It is basically a linear RFQ completely bent on a circle. In this paper, equations of motion are derived and solved for particles circulating and accelerated in the device. Comparison of beam performance is made with that of conventional storage and accelerator rings. The advantages are: very small beam dimensions, high beam intensity, and considerably less susceptibility to errors, which makes the motion of particles in the device very stable. We first discuss the use of the CRFQ as a storage ring; in the second part of the paper, we investigate also the possibility of acceleration. The design of several applications is discussed, including, a medical accelerator and a synchrotron radiation source. Both are made of a structure much more compact than that of other conventional rings.

\section{Introduction}

In this report we describe a novel idea of storage and accelerator ring for intense and dense beams of light and heavy ions, protons, and electrons in a more compact structure. The concept takes advantage of established principles $[1,2]$ of operation of conventional accelerators, ion traps, and RFQ. The proposed new storage ring is basically a circular RFQ bent completely on a circle, and closed mechanically. Focusing the particle motion is derived from the RF field of the structure, thus eliminating the need of quadrupole magnets. Actually, similarly to the linear RFQ, the focusing is continuous in the CRFQ, with no interruption, and thus is expected to be more effective than that of conventional storage rings, where the focusing action is localized in quadrupoles placed at some distance from each other. As a consequence, a more compact structure is also expected. In the case acceleration is not required, but only storage of particles, the four rods of the CRFQ, equivalent to those of the linear RFQ, are smooth, with no convolutions shaped internally. Care can then be taken so that the ratio of the CRFQ circumference to the focusing period length is not an integral number. In this case, electrically the structure is not closed on itself and, to a particle traveling inside, it looks like a long unbounded transport. For these reasons, similar to those used in a linear RFQ, it is expected that particle beams can be stored at intensities and densities higher than those achieved in conventional storage rings. Also, for the same reasons, one would expect less susceptibility to errors and imperfections, making the particle motion more stable. It will be shown that for a very low particle velocity $(\beta<<1)$ the particle motion is bent on a circle without the help of an external bending field. The bending is provided by the focusing action of the RF forces that create a reference distorted-orbit displaced

\footnotetext{
* Work performed under the auspices of the US Department of Energy
} 
horizontally and outward. In case the amount of the displacement is small compared to the separation between the rods there is no need of an external bending field. But at larger velocities the displacement increases considerably, for reasonable RF parameters, and an external bending magnet is required to confine the beam within the aperture of the RF structure. In this case the CRFQ is placed entirely in the gap of an external unbroken dipole magnet that covers the entire circumference of the ring, and still results in a very compact device. Under these conditions, the CRFQ can be used as a storage ring for any kind of particle and, in principle, for any energy.

The lack of periodicity avoids the appearance of resonances between the particle oscillation frequency and the revolution frequency. It is then possible to cross a large range of oscillation frequency without disrupting the stability of the particle motion. In particular it is possible as in the linear RFQ to depress considerably the focusing with the space-charge forces, much more than it is possible in conventional storage and accelerator rings. The focusing period is given by the product of the particle velocity with the RF wavelength and can be made very short; much shorter than it is possible in conventional magnetic rings. Correspondingly also the amplitude-lattice function is reduced and, with a given beam emittance, the transverse beam size is also smaller. These two features, higher intensity and smaller beam dimension, combined together will allow very high beam brilliance.

The CRFQ does not have to be necessarily exactly circular. Long straight sections can be introduced, and the special case of a race-track-shaped CRFQ is discussed in the paper. In this case a geometrical periodicity appears but nonetheless it does not seem to have consequences similar to those of conventional rings.

It is also possible to accelerate all types of particles in the CRFQ. For this purpose, the rods have a convolution shaped on their facing side. The length of the convolution, that generates a longitudinal electric field, equals the focusing period. It is then required that the number of focusing periods per turn is exactly an integral number. It is not clear whether this will cause the appearance of resonances, and what their effect on the particle motion stability is. During acceleration the particle velocity will vary, and this variation has to be compensated with an increase of the RF frequency so that the period length is preserved during the acceleration cycle. In the acceleration mode, an external bending magnet is always required that needs also to be ramped with the beam momentum.

The paper starts with the presentation of the CRFQ geometry and definitions. After deriving the RF fields acting on the particle, the equations of motion are obtained and solved in the linear approximation. First we discuss the case without acceleration where the four rods are smooth and do not present convolution. It is found that transverse motion is confined by the oscillating behavior of the RF forces in the similar fashion of conventional alternating gradient accelerators. Thus, concepts describing the particle motion, similar to those of conventional accelerators, can still be used. A new concept appears that deals with the displacement of the reference orbit. The momentum dependence of the usual lattice parameters is also estimated. It is found that dispersion and chromaticity have in the CRFQ a behavior well different from that of conventional 
accelerators. A race-track-shaped CRFQ with two long straights inserted is also investigated. Finally, acceleration and how the rods need to be convoluted, to generate the required longitudinal electric field, are also studied. The procedure is similar to that of the linear RFQ. Both transverse and longitudinal space-charge limits are evaluated, again following closely the same approach used for the conventional linear RFQ.

Though the applications of the CRFQ storage and accelerator ring can be numerous, we have offered in the last part of the paper six examples: a CRFQ prototype, a modest ring for the demonstration of the operation principles; a low-energy ion storage ring; a lowenergy proton beam storage ring for the production of gamma and neutron radiation; a compact medical accelerator; a high-energy proton accelerator, and a similar electron accelerator; and, finally, a compact light source CRFQ storage ring. There are some technical outstanding issues that need to be solved for the demonstration of the CRFQ ring. Among them, it is important to learn how to input RF power in the device and how to inject and to extract a particle beam.

\section{The Circular Radio-Frequency Quadrupole Storage Ring}

The Circular Radio-Frequency Quadrupole (CRFQ) storage ring is composed of four concentric annular metallic rods made, for instance, of copper. Figure 1 shows the plane view (a) and the cross-section (b) of the device. The major radius $\mathrm{R}$ joins the center $\mathrm{O}$ on the mid-plane to the point $P$ equidistant from the four rods. Each rod has a circular crosssection. The inner diagonal separation between rods is $2 \mathrm{~b}$. It is assumed that the major radius $\mathrm{R}$ is considerably larger than the minor radius $\mathrm{b}$, that is $\mathrm{R}>>\mathrm{b}$. The parallel and up-and-down arrangement of the coils, as shown in Figure 1, is suggested to make injection of the beam easier, without encountering obstructions, and for the insertion of localized items like rf cavities, vacuum pumps and beam diagnostic components.

(a)

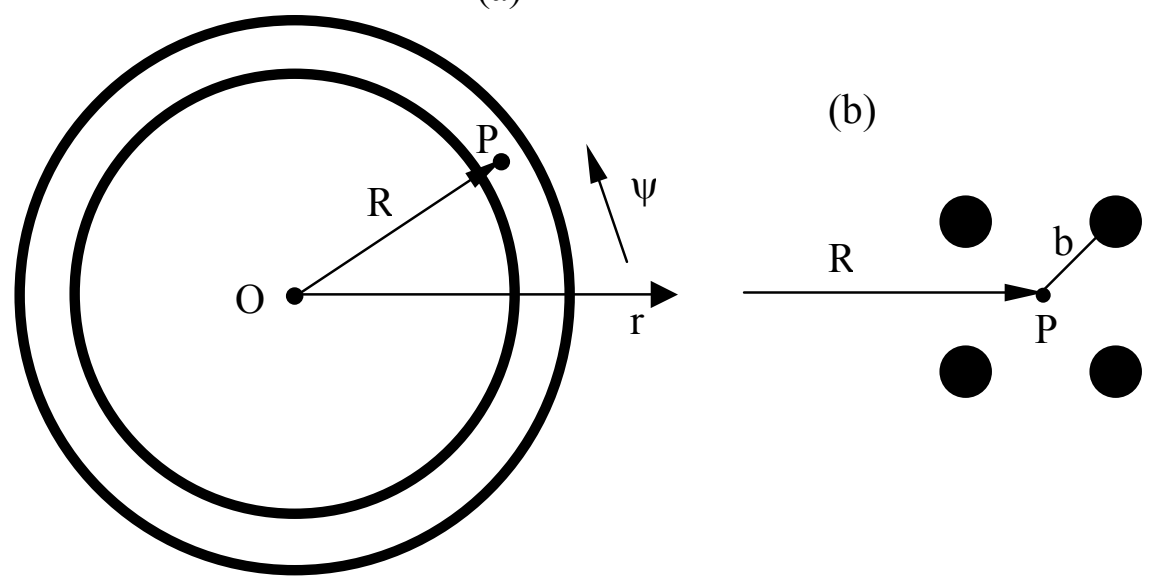

Figure 1. Plan view (a) and cross-section (b) of the CRFQ

A cylindrical coordinate system $(\mathrm{r}, \psi, \mathrm{z})$ is employed; with the radial coordinate $\mathrm{r}$ measured on the mid-plane from the origin $\mathrm{O}$. The vertical axis $\mathrm{z}$ is perpendicular to the 
plane view (a), goes through the origin $\mathrm{O}$, and is oriented upward. The angular coordinate is $\psi$. A more useful coordinate system is the toroidal system $(\eta, \theta, \psi)$ [3]. The angular coordinate $\psi$ coincides with the coordinate of the same symbol in the cylindrical system. The toroidal surface is described by the coordinate $\eta$, with $\eta=0$ the vertical axis through the origin $\mathrm{O}$, and $\eta=\infty$ the circle ${ }^{*}$ of radius $\mathrm{R}$ going through $\mathrm{P}$. The coordinate $\theta$ is the azimuth around the toroidal surface, rotating clockwise, with the origin $\theta=0$ on the midplane, pointed inward. To solve the associated Maxwell's equations, it is more convenient to consider a reduced toroidal coordinate system. This is obtained by replacing $\eta$ with the local toroidal variable $\rho=\left(x^{2}+z^{2}\right)^{1 / 2}$. Here $x=r-R$ is the radial distance on the mid-plane from the circumference of radius $R$. It is $\rho=0$ on the same circumference, and $\rho=b$ on the toroidal surface touching the inner part of the four rods (see Figure 2).

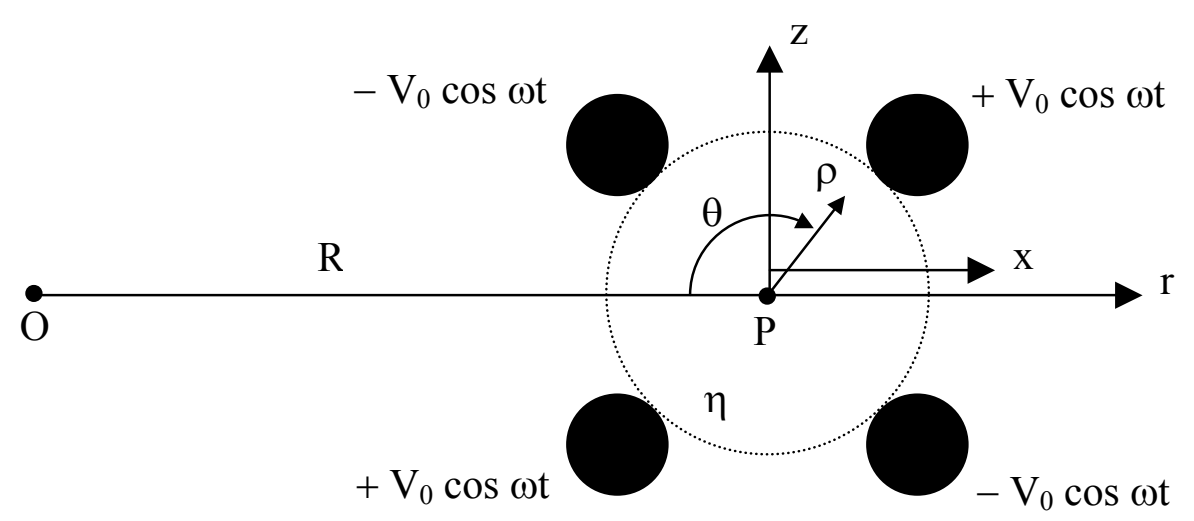

Figure 2. Four-Rods and Toroidal Coordinate System

\section{Field Expansion}

The rods are placed at alternating RF voltages as shown in Figure 2. The peak voltage is $\mathrm{V}_{0}$ and the angular frequency of the excitation is $\omega=2 \pi \mathrm{f}$, to which wavelength $\lambda=\mathrm{c} / \mathrm{f}$ is associated, with $\mathrm{c}$ the speed of light. Aside from the variation with time, the voltage is otherwise constant along the length of the rods. Apart from the term $\cos (\omega t)$ which shows explicitly the time dependence, let $\mathrm{V}(\rho, \theta)$ be the scalar voltage distribution in the region of the CRFQ within the four rods. Because of the assumed symmetry, there is no dependence on the angular coordinate $\psi$. The wave equation satisfied by $\mathrm{V}(\rho, \theta)$ is

$\nabla^{2} \mathrm{~V}(\rho, \theta)+\mathrm{q}^{2} \mathrm{~V}(\rho, \theta)=0$,

where $\mathrm{q}=\omega / \mathrm{c}=2 \pi / \lambda$. The Laplacian should be expressed in the main toroidal coordinate system, but the resulting second-order differential equation is too complex and difficult to solve. We then rely on the assumption $\mathrm{R}>>\mathrm{b}$, and work out an expansion

\footnotetext{
* The actual location of the geometrical center P, equidistant from the four coils, is displaced outward from the $\eta=\infty$ circle by a small amount $b^{2} / 2 R$.
} 
which gives an approximate representation in the region surrounded by the four rods, that is for $\rho<$ b. Following the details of the computation shown in Appendix A,

$\rho^{2} \partial^{2} \mathrm{~V} / \partial \rho^{2}+\rho \partial \mathrm{V} / \partial \rho+\partial^{2} \mathrm{~V} / \partial \theta^{2}+\mathrm{q}^{2} \rho^{2} \mathrm{~V}=0$

Observe that this equation does not depend explicitly on the major radius $\mathrm{R}$, but it does implicitly through the definition of the reduced toroidal coordinate system. The solution of Eq. (2) can be expressed in terms of Bessel functions,

$V(\rho, \theta)=\Sigma_{n} a_{n} J_{n}(q \rho) \sin (n \theta) \cos (\omega t)$

satisfying, from symmetry considerations, the condition $\mathrm{V}=0$ on the mid-plane.

Similarly, there is also a vector potential A. The relationship of these potential functions to the field components is given in Appendix B. It is sufficient to retain only the lowestorder term $n=2$, and finally, after expansion of the Bessel function, we have

$$
\begin{aligned}
\mathrm{V}(\rho, \theta) & =\mathrm{a}_{2} \mathrm{~J}_{2}(\mathrm{q} \rho) \sin (2 \theta) \cos (\omega \mathrm{t}) \\
& =\mathrm{V}_{0}(\rho / \mathrm{b})^{2} \sin (2 \theta) \cos (\omega \mathrm{t}) \\
& =-2 \mathrm{~V}_{0}\left(\mathrm{x} \mathrm{z} / \mathrm{b}^{2}\right) \cos (\omega \mathrm{t})
\end{aligned}
$$

where $\mathrm{x}=-\rho \cos \theta, \mathrm{z}=\rho \sin \theta$. From this we can derive the radial and vertical electric field components, as shown in Appendix B,

$$
\begin{aligned}
& \mathrm{E}_{\mathrm{x}}=2 \mathrm{~V}_{0}\left(\mathrm{z} / \mathrm{b}^{2}\right) \cos (\omega \mathrm{t}) \\
& \mathrm{E}_{\mathrm{z}}=2 \mathrm{~V}_{0}\left(\mathrm{x} / \mathrm{b}^{2}\right) \cos (\omega \mathrm{t})
\end{aligned}
$$

It is to be noticed the inversion of reciprocity between field components and point coordinates. This is due to the chosen mutual arrangement of the four rods, shown in the cross-section view (b) of Figure 1. There is also a solenoidal magnetic field directed along the main direction of the beam motion. Its expression is given in Appendix B. The magnitude of this field is though small, and can be ignored.

\section{Equations of Motion}

The particle beam circulates in the CRFQ storage ring between the four rods along the circumference of radius $\mathrm{R}$, on the mid-plane. We shall use the cylindrical coordinate system $(r, \psi, z)$ and the reduced coordinate $x=r-R$. The equations of motion of a single particle can be derived from the Lagrangian

$$
\mathcal{L}=-\mathrm{Am} \mathrm{c}^{2}\left(1-\beta^{2}\right)^{1 / 2}-\mathrm{Q} \text { e }[\mathrm{V}(\mathrm{x}, \mathrm{z})-\mathbf{v} \cdot \mathbf{A} / \mathrm{c}]
$$

where $\mathrm{m}$ is the mass at rest of a proton, e the electron electric charge, $\mathrm{Q}$ the charge state, and $A$ the mass number of the ion. $\beta$ and $\gamma$ are respectively the usual velocity and energy relativistic factors. By denoting $\mathrm{v}_{\mathrm{r}}=\mathrm{dr} / \mathrm{dt}, \mathrm{v}_{\psi}=\mathrm{d} \psi / \mathrm{dt}$, and $\mathrm{v}_{\mathrm{z}}=\mathrm{dz} / \mathrm{dt}$, it is 
$c^{2} \beta^{2}=v_{r}^{2}+r^{2} v_{\psi}^{2}+v_{z}^{2}$. The vector potential $\mathbf{A}$ may also include the contribution from an external bending field $B_{\text {ext }}$, in which case $\mathbf{A}=\left[0,-\mathrm{rB}_{\text {ext }} / 2,0\right]$. We shall ignore the contribution of the excitation RF field to the potential vector $\mathbf{A}$, because, as it is shown in Appendix A, it is of higher order. In linear approximation, the RF field is derived only from the scalar potential V(x, z), Eq. (4), and is given by Eq.s (5 and 6).

The Lagrangian equations of motion are

$\mathrm{d}\left(\partial \mathcal{L} / \partial \mathrm{v}_{\mathrm{r}}\right) / \mathrm{dt}=\partial \mathcal{L} / \partial \mathrm{r}$

and similarly for the other equations obtained by replacing $\mathrm{r}$ with $\mathrm{x}, \psi$ and $\mathrm{z}$. We have

$\operatorname{Amd}\left(\gamma \mathrm{v}_{\mathrm{r}}\right) / \mathrm{dt}=\mathrm{Am} \gamma \mathrm{r}(\mathrm{d} \psi / \mathrm{dt})^{2}-\mathrm{Q}$ e $\partial \mathrm{V} / \partial \mathrm{r}-\mathrm{Qe} \mathrm{r}(\mathrm{d} \psi / \mathrm{dt}) \mathrm{B}_{\mathrm{ext}} / \mathrm{c}$

$\operatorname{Amd}\left(\gamma \mathrm{v}_{\mathrm{z}}\right) / \mathrm{dt}=-\mathrm{Q}$ e $\partial \mathrm{V} / \partial \mathrm{z}$

It is seen in particular that the generalized angular momentum

$\mathrm{P}_{\psi}=\operatorname{Am} \gamma \mathrm{r}^{2}(\mathrm{~d} \psi / \mathrm{dt})-\mathrm{Qe} \mathrm{r}^{2} \mathrm{~B}_{\mathrm{ext}} / 2 \mathrm{c}$

is a constant of motion. In the case of storage mode that is being considered here, the particle energy does not change, and the relativistic factors $\beta$ and $\gamma$ can be treated as constant ${ }^{*}$. We shall also introduce the angular revolution frequency $\omega_{0}=\mathrm{d} \psi / \mathrm{dt} \approx \beta \mathrm{c} / \mathrm{r}$. The last two equations can then be written as follows

$\mathrm{d}^{2} \mathrm{x} / \mathrm{d} \mathrm{t}^{2}-\left(2 \mathrm{Q}\right.$ e $\left.V_{0} / A \gamma m b^{2}\right) \mathrm{z} \cos (\omega \mathrm{t})=\mathrm{r} \omega_{0}^{2}-\mathrm{Qe} \beta \mathrm{B}_{\text {ext }} / \mathrm{A} \gamma \mathrm{m}$

$\mathrm{d}^{2} \mathrm{z} / \mathrm{d} \mathrm{t}^{2}-\left(2 \mathrm{Q}\right.$ e $\left.\mathrm{V}_{0} / \mathrm{A} \gamma \mathrm{m} \mathrm{b}^{2}\right) \mathrm{x} \cos (\omega \mathrm{t})=0$

We need to make one more transformation from time $t$ to path length $\mathrm{s}=\beta \mathrm{ct}$. Moreover, $\omega \mathrm{t}=\mathrm{ks}$ with

$\mathrm{k}=2 \pi / \beta \lambda=2 \pi / \mathrm{L}$

where $\mathrm{L}$ is the focusing period. With these transformations Eq.s (12 and 13) become

$\mathrm{d}^{2} \mathrm{x} / \mathrm{ds^{2 }}-\left(2 \mathrm{Q}\right.$ e $\left.\mathrm{V}_{0} / \mathrm{A} \gamma \mathrm{m} \mathrm{c}^{2} \beta^{2} \mathrm{~b}^{2}\right) \mathrm{z} \cos (\mathrm{ks})=1 / \mathrm{r}-1 / \mathrm{r}_{\mathrm{L}}$

$\mathrm{d}^{2} \mathrm{z} / \mathrm{d} \mathrm{s}^{2}-\left(2 \mathrm{Q}\right.$ e $\left.\mathrm{V}_{0} / \mathrm{A} \gamma \mathrm{m} \mathrm{c} \mathrm{c}^{2} \beta^{2} \mathrm{~b}^{2}\right) \mathrm{x} \cos (\mathrm{ks})=0$

where

$r_{L}=A \gamma m \beta c^{2} /$ Qe $B_{\text {ext }}$

\footnotetext{
${ }^{*}$ In reality, under the effect of the electric RF field that does do work, the particle energy oscillates at the same excitation frequency $\omega$ but with an amplitude not exceeding $2 \mathrm{QeV}_{0} \mathrm{xz} / \mathrm{b}^{2}$.
} 
is the Lorenz radius. Eq.s (15 and 16) are those of coupled linear oscillators under the action of a periodic restoring force with period $\mathrm{L}=\beta \lambda$.

It is convenient at this point to introduce the RFQ parameter [4]

$\mathrm{B}=2 \mathrm{Qe} \mathrm{V}_{0} \lambda^{2} / \mathrm{A} \gamma \mathrm{mc}^{2} \mathrm{~b}^{2}$

and we have

$\mathrm{d}^{2} \mathrm{x} / \mathrm{d} \mathrm{s} \mathrm{s}^{2}-\left(\mathrm{B} / \mathrm{L}^{2}\right) \mathrm{z} \cos (\mathrm{ks})=1 / \mathrm{r}-1 / \mathrm{r}_{\mathrm{L}}$

$\mathrm{d}^{2} \mathrm{z} / \mathrm{d} \mathrm{s}^{2}-\left(\mathrm{B} / \mathrm{L}^{2}\right) \mathrm{x} \cos (\mathrm{ks})=0$

At the r. h. side of Eq. (19), taking $x<<R$, and after expansion, we have $1 / r=1 / R-$ $x / R^{2}$. The second term $x / R^{2}$ adds the focusing from curvature to the total focusing on the mid-plane. This is the equivalent of the curvature effect encountered in bending magnets of more conventional storage rings. As long as $\mathrm{R}^{2}>>\mathrm{L}^{2}$ this term can be ignored, and we shall assume here that this is always the case. At the r. h. side of Eq. (19) we shall keep only the term $1 / \mathrm{R}-1 / \mathrm{r}_{\mathrm{L}}$. In absence of an external bending field $\mathrm{B}_{\text {ext }}=0$ and $1 / \mathrm{r}_{\mathrm{L}}=0$. At one extreme, the term at the r.h. side of Eq. (19) can be cancelled entirely by setting the external bending field so that $r_{L}=R$. Other intermediate values are of course also possible, with only a partial cancellation, if desired, of the curvature term at the r.h. side of Eq. (19).

The coupling between the two components of motion can be resolved by transforming to

$\mathrm{u}=\mathrm{x}+\mathrm{z}$

$\mathrm{v}=\mathrm{x}-\mathrm{z}$

which satisfy the decoupled equations

$\mathrm{d}^{2} \mathrm{u} / \mathrm{d} \mathrm{s}^{2}-\left(\mathrm{B} / \mathrm{L}^{2}\right) \mathrm{u} \cos (\mathrm{ks})=1 / \mathrm{R}-1 / \mathrm{r}_{\mathrm{L}}=1 / \underline{\mathrm{R}}$

$\mathrm{d}^{2} \mathrm{v} / \mathrm{d} \mathrm{s}^{2}+\left(\mathrm{B} / \mathrm{L}^{2}\right) \mathrm{v} \cos (\mathrm{ks})=1 / \mathrm{R}-1 / \mathrm{r}_{\mathrm{L}}=1 / \underline{\mathrm{R}}$

These equations show that the two modes of oscillation do not coincide with the $\mathrm{x}$ - and $\mathrm{z}$ axis, but have directions obtained by rotating them by $45^{\circ}$. Since an ensemble of particles can be represented in the real space as an ellipse, the actual beam cross-section breaths along the CRFQ axis as shown in Figure 3, in the way we are going to estimate below.

\section{Momentum Dependence}

Eqs. (23 and 24) are identical, apart from a sign. The solution of one can be derived from the other by a shift of $180^{\circ}$. We shall, therefore, concentrate on the solution of Eq. (23).

Particles of a beam have a spread of momentum values. The CRFQ is in particular designed with a reference momentum value in mind that we shall refer to as $\mathrm{p}_{\mathrm{s}}$. All 
parameters associated to the reference particle will be denoted with the subscript s. In particular the velocity $\beta_{\mathrm{s}} \mathrm{c}$ corresponds to this. All other particles have a momentum value $\mathrm{p}=\mathrm{p}_{\mathrm{s}}(1+\delta)$, where $\delta<<1$ is the relative momentum deviation. The parameters associated to any other particle will be denoted with the same symbol but without any subscript. In particular the velocity is $\beta \mathrm{c}$. By differentiating $\beta=\beta_{\mathrm{s}}\left(1+\delta / \gamma_{\mathrm{s}}^{2}\right)$, $\gamma=\gamma_{\mathrm{s}}\left(1+\beta_{\mathrm{s}}{ }^{2} \delta\right)$, and $\beta \gamma=\beta_{\mathrm{s}} \gamma_{\mathrm{s}}(1+\delta)$.

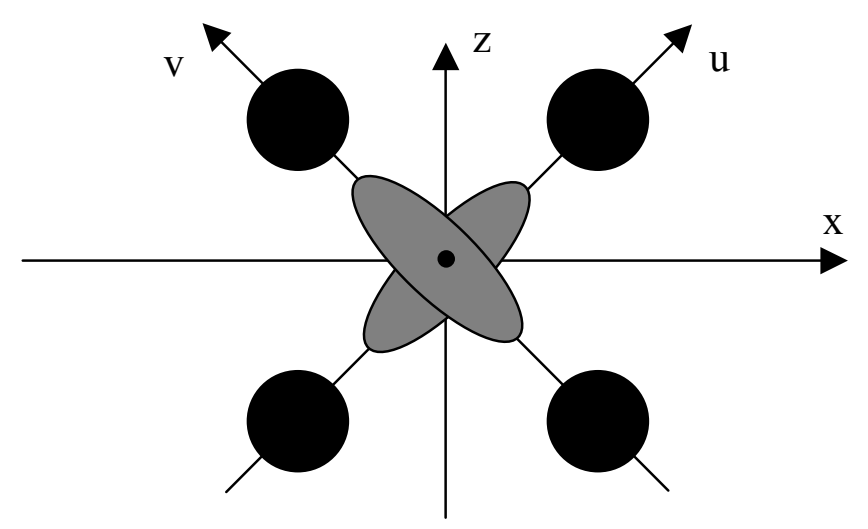

Figure 3. Normal Modes of oscillations in the CRFQ

As written, Eq. (23) applies to any particle with momentum p. It is seen that the momentum dependence enters through the velocity $\beta \mathrm{c}$ in the period of the external focusing $L=L_{s}\left(1+\delta / \gamma_{s}^{2}\right)$, where $L_{s}=\beta_{s} \lambda$ is the period associated to the reference momentum value. There are two places in Eq. (23) where this occurs. First, in the amplitude $\mathrm{B} / \mathrm{L}^{2}$ and, then, in the frequency $\mathrm{k}$ of the restoring force. In both cases, the dependence is through the period $\mathrm{L}$ and the parameter $\mathrm{B}$. The radius $\mathrm{R}$ does not exhibit momentum dependence, but the Lorenz radius $\mathrm{r}_{\mathrm{L}}$ does. Thus we have

$$
\begin{array}{ll}
\mathrm{B} / \mathrm{L}^{2} & =\left(\mathrm{B}_{\mathrm{s}} / \mathrm{L}_{\mathrm{s}}{ }^{2}\right)\left[1-\left(2-\beta_{\mathrm{s}}{ }^{2}\right) \delta\right] \\
\mathrm{k} & =\mathrm{k}_{\mathrm{s}}\left(1-\delta / \gamma_{\mathrm{s}}{ }^{2}\right) \\
\mathrm{r}_{\mathrm{L}} & =\mathrm{r}_{\mathrm{Ls}}(1+\delta)
\end{array}
$$

This result shows a significant departure from the behavior in a conventional storage ring with magnets. There is a different, stronger momentum dependence on the strength of the focusing. The focusing periodicity varies with momentum, whereas in a conventional storage ring it is absolutely constant. Finally, there is a mixed momentum dependence in the curvature term as opposed to the case of a conventional storage ring.

\section{Solution of the Equation of Motion}

Let us solve Eq. (23) for a particle with reference momentum $p_{s}$. The equation is nonhomogenous because of the curvature term at the $r$. $h$. side. Let us study first the homogenous equation

$$
d^{2} \mathrm{u} / \mathrm{ds}^{2}-\left(\mathrm{B}_{\mathrm{s}} / \mathrm{L}_{\mathrm{s}}{ }^{2}\right) \mathrm{u} \cos \left(2 \pi \mathrm{s} / \mathrm{L}_{\mathrm{s}}\right)=0
$$


The solution can be described in terms of Mathieu functions (see Appendix D), but it can also be treated on the computer, and numerically solved [5]. In analogy to FODO cells of conventional storage rings with magnets [6], the solution is a betatron oscillation described by a local amplitude function $\beta_{\mathrm{Ls}}$, which varies periodically with the path length $\mathrm{s}$ and has the same periodicity $\mathrm{L}_{\mathrm{s}}$ of the focusing, and a phase advance $\mu_{\mathrm{s}}$ per period. For instance, a phase advance per period of $90^{\circ}$ is achieved by setting the RF peak voltage $V_{0}$ so that $B_{s}=6.80928$. At the same time the amplitude lattice function $\beta_{L s}$ varies between a minimum $0.905 \mathrm{~L}_{\mathrm{s}}$ and a maximum $1.878 \mathrm{~L}_{\mathrm{s}}$, with an average value of about $1.39 \mathrm{~L}_{\mathrm{s}}$ (see Figure 4). The ratio of the extreme values of the amplitude function is 2.075. Since the beam dimension is given locally by the square root of $\beta_{\mathrm{Ls}}$, it is seen that, for a phase advance per period of $90^{\circ}$, the relative maximum change of the beam size is $44 \%$. If along one mode of oscillation ( $\mathrm{u}$ or $\mathrm{v}$ ) the beam size is the largest, than it is the smallest along the other mode, and vice-versa. The beam profile is usually an ellipse with a shape oscillating between the two extremes, as shown in Figure 3. A complete envelope oscillation takes place during one focusing period. The other quantity which describes the particle motion is the ellipse rotation parameter $\alpha_{\mathrm{Ls}}$ related to the amplitude function by $\mathrm{d} \beta_{\mathrm{Ls}} / \mathrm{d} \mathrm{s}=-2 \alpha_{\mathrm{Ls}}$. This quantity vanishes at the extremes of $\beta_{\mathrm{Ls}}$. Calculated in the middle of a period, where it is the largest in absolute value, it does not depend on the period length and it is $\alpha_{\mathrm{Ls}}= \pm 1.516$.

The following trigonometric expansion of the amplitude function applies for a $90^{\circ}$ phase advance per period $\left(\mathrm{k}_{\mathrm{s}} \mathrm{s}=2 \theta\right)$

$\beta_{\mathrm{Ls}} / \mathrm{L}_{\mathrm{s}}=\mathrm{a}_{0}-\mathrm{a}_{1} \cos (2 \theta)+\mathrm{a}_{2} \cos (4 \theta)+\ldots$

with $\mathrm{a}_{0}=1.3600, \mathrm{a}_{1}=0.4865, \mathrm{a}_{2}=0.0315$ and a residual error not exceeding \pm 0.0016 .

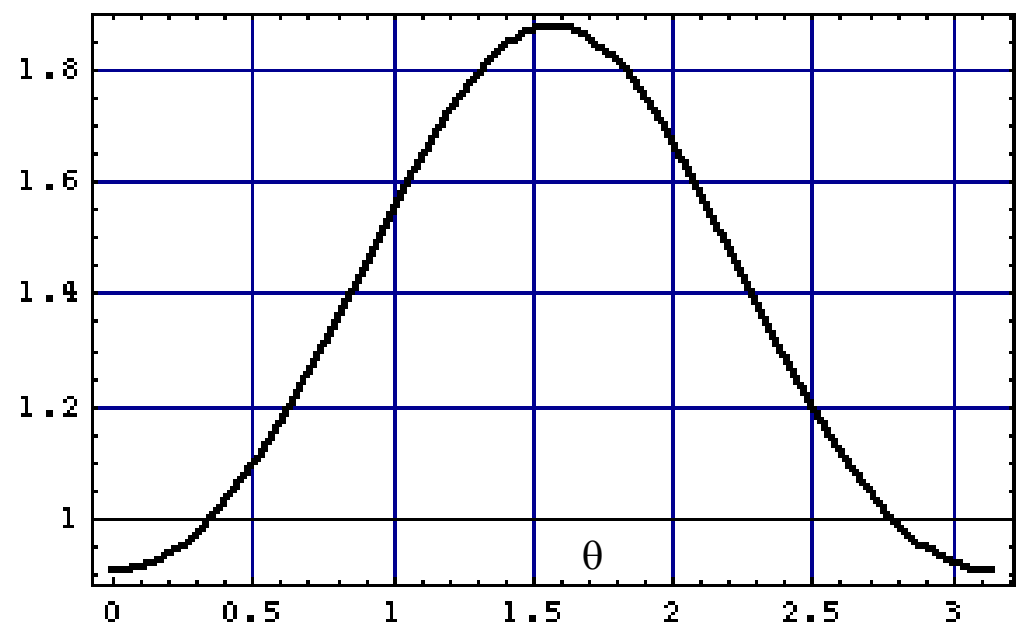

Figure 4. The amplitude lattice function $\beta_{\mathrm{Ls}} / \mathrm{L}_{\mathrm{s}}$ over one focusing period $(0-\pi)$. 


\section{Orbit Distortion}

The general solution of Eq. (23) is the sum of a particular solution $\underline{u}(\mathrm{~s})$ of the inhomogeneous equation, which we take here to be periodic with the same periodicity L of the focusing period, and of the "free betatron oscillation" solution that is the solution of the associated homogenous equation we have studied in the previous section. With the variable replacement $2 \theta=\mathrm{ks}$.

$\underline{\mathrm{u}}(\theta)=\mathrm{w}(\theta) \mathrm{L}^{2} / \pi^{2} \underline{\mathrm{R}}$

where $\mathrm{w}(\theta)$ is an oscillatory universal function, with a similar behavior of $\beta_{\mathrm{L}}$, shown in Figure 5. For a phase advance of $90^{\circ}$ per period, it varies between $\mathrm{w}_{\min }=13.942$ and $\mathrm{w}_{\max }=19.743$. Actually, it can be shown that, with an error not larger than $\pm 1 \%$,

$\mathrm{W}=14.5\left(\beta_{\mathrm{L}} / \mathrm{L}\right)^{1 / 2}$.

Also,

$\mathrm{W}=\mathrm{w}_{0}-\mathrm{w}_{1} \cos (2 \theta)+\mathrm{w}_{2} \cos (4 \theta)+\ldots$

with $\mathrm{w}_{0}=16.7758, \mathrm{w}_{1}=2.8995, \mathrm{w}_{2}=0.0625$ and a residual error not exceeding \pm 0.0010 .

Thus, for every particle there is an orbit distortion caused by the curvature of the CRFQ, around which open betatron oscillations, of the type described in the previous section, are performed. The orbit distortion is given by Eq. (30). Since $\underline{\mathrm{R}}>>\mathrm{L}$, the distortion is typically a small fraction of the period $\mathrm{L}$.

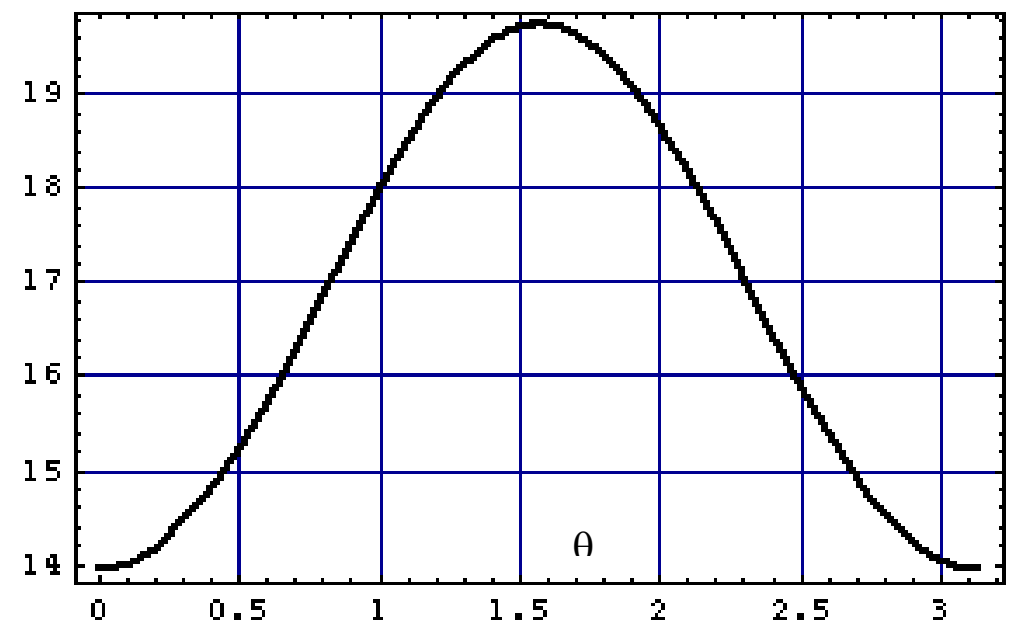

Figure 5. The scaled dispersion function $\mathrm{w}(\theta)$ over one focusing period $(0-\pi)$

Orbit distortion appears for both the rotated coordinates $u$ and $v$. But the minima on one plane correspond to the maxima on the other; that is the waveforms are shifted by $180^{\circ}$ 
per period with respect to each other. Recalling the coordinate definition by Eqs. (21 and 22), it is seen that the terms in Eq. (32) proportional to $\mathrm{w}_{0}$ and $\mathrm{w}_{2}$ contribute to the orbit distortion and dispersion on the horizontal (x) direction, whereas the term in $\mathrm{w}_{1}$ contributes on the vertical (z) direction.

Let $2 \pi R_{c}$ denote the path length of a particle trajectory after one complete revolution. The number of periods traveled during that length is $n=2 \pi R_{c} / L$. This number can be very large and, usually, has a non-integral value. This feature does not exist in conventional storage rings that have integral and low periodicity. To determine the one-turn path length we have to perform the following operation

$2 \pi R_{c}=2 \pi R\left[1+\left(L^{3} / 2 \pi^{4} R^{2} \underline{R}\right) \int w(\theta) d \theta\right]$

where the integral is taken over one complete revolution. In good approximation, since $\mathrm{n} \gg \mathrm{1}$,

$\int \mathrm{w}(\theta) \mathrm{d} \theta=\mathrm{n} \pi<\mathrm{W}>$

with $<w>$ the average value of $w(\theta)$ over one period (from 0 to $\pi$ ). By combining the equations above

$\mathrm{R}_{\mathrm{c}}=\mathrm{R}\left(1+\mathrm{wW}_{\mathrm{W}} \mathrm{L}^{2} / \pi^{2} \mathrm{R} \underline{\mathrm{R}}\right)$

For a $90^{\circ}$ phase advance per period $\langle\mathrm{w}\rangle=\mathrm{w}_{0}=16.78$. In all cases, the difference $\Delta \mathrm{R}=\mathrm{R}_{\mathrm{c}}-\mathrm{R}=\mathrm{w}_{0} \mathrm{~L}^{2} / \pi^{2} \underline{\mathrm{R}}$ is only a small fraction of the period length $\mathrm{L}$, as long as $\mathrm{L}<<\underline{\mathrm{R}}$.

There are two extreme cases: (i) No external bending field, $B_{\text {ext }}=0$, in which case $\underline{R}=R$ and $1 / r_{L}=0$. The main trajectory also oscillates according to $\mathrm{w}(\mathrm{s})$, but does not close, as long the number of periods $\mathrm{n}$ is not integral. (ii) The external field is adjusted so that $\mathrm{r}_{\mathrm{L}}=\mathrm{R}$ and $1 / \underline{\mathrm{R}}=0$, in which case $\Delta \mathrm{R}=0$ identically. The main trajectory closes exactly after one turn and coincides with the circle of radius $R$.

For the particle with reference momentum $\mathrm{p}_{\mathrm{s}}$, the length of the orbit after one turn is $\mathrm{R}_{\mathrm{cs}}=\mathrm{R}\left(1+{ }_{\mathrm{w}}>\mathrm{L}_{\mathrm{s}}{ }^{2} / \pi^{2} \mathrm{R} \underline{\mathrm{R}}_{\mathrm{s}}\right)$. The revolution frequency is $\mathrm{f}_{\mathrm{s}}=2 \pi \mathrm{R}_{\mathrm{cs}} / \mathrm{c} \beta_{\mathrm{s}}$. For any other particle it is $f=2 \pi R_{c} / c \beta$. Let $\mu$ be the phase advance per period; we can introduce the "betatron tune" $v=n \mu / 2 \pi$, that is the number of free (betatron) oscillations per revolution. Moreover, the betatron frequency is $f_{\beta}=f v$. For the particle with reference momentum $\mathrm{p}_{\mathrm{s}}$, the number of periods per revolution $\mathrm{n}_{\mathrm{s}}=2 \pi \mathrm{R}_{\mathrm{cs}} / \mathrm{L}_{\mathrm{s}}$, the phase advance per period is $\mu_{\mathrm{s}}$, the betatron tune $v_{\mathrm{s}}=\mathrm{n}_{\mathrm{s}} \mu_{\mathrm{s}} / 2 \pi$, and the betatron frequency $\mathrm{f}_{\beta \mathrm{s}}=\mathrm{f}_{\mathrm{s}} v_{\mathrm{s}}$.

\section{Dispersion}

We have seen that the orbit distortion is given by Eq.(30). After extracting the linear dependence with the momentum error $\delta$, we have 
$\underline{\mathrm{u}}(\theta)=\underline{\mathrm{u}}_{\mathrm{s}}(\theta)+\left(\mathrm{L}_{\mathrm{s}} / \pi\right)^{2} \mathrm{w}_{\mathrm{s}}(\theta)\left[\left(2 / \gamma_{\mathrm{s}}{ }^{2} \underline{\mathrm{R}}_{\mathrm{s}}\right)+\left(1 / \mathrm{r}_{\mathrm{Ls}}\right)-\chi_{\mathrm{w}}(\theta) \beta_{\mathrm{s}}{ }^{2} / \underline{\mathrm{R}}_{\mathrm{s}}\right] \delta$

where

$\underline{\mathrm{u}}_{\mathrm{s}}(\theta)=\mathrm{L}_{\mathrm{s}}{ }^{2} \mathrm{~W}_{\mathrm{s}}(\theta) / \pi^{2} \underline{\mathrm{R}}_{\mathrm{s}}$

is the orbit distortion for the particle with momentum $p_{s}, w_{s}(\theta)$ is the central value of the universal function $\mathrm{w}(\theta)$ estimated for the RFQ parameter $\mathrm{B}=\mathrm{B}_{\mathrm{s}}, 1 / \underline{\mathrm{R}}_{\mathrm{s}}=1 / \mathrm{R}-1 / \mathrm{r}_{\mathrm{Ls}}$, and

$\chi_{\mathrm{w}}(\theta)=\left(\mathrm{B}_{\mathrm{s}} / \mathrm{w}_{\mathrm{s}}\right) \partial \mathrm{w} / \partial \mathrm{B}$

We define the dispersion $\eta(\theta)$ as the variation of the orbit distortion with the momentum error $\delta$, that is

$\eta(\theta)=\left(\mathrm{L}_{\mathrm{s}} / \pi\right)^{2} \mathrm{w}_{\mathrm{s}}(\theta)\left[\left(2 / \gamma_{\mathrm{s}}{ }^{2} \underline{\mathrm{R}}_{\mathrm{s}}\right)+\left(1 / \mathrm{r}_{\mathrm{Ls}}\right)-\chi_{\mathrm{w}}(\theta) \beta_{\mathrm{s}}{ }^{2} / \underline{\mathrm{R}}_{\mathrm{s}}\right]$

As for the length of the orbit distortion, also the contribution to the dispersion in the horizontal plane ( $\mathrm{x}$ ) is given by the $\mathrm{w}_{0}$ and $\mathrm{w}_{2}$ terms of the expansion Eq. (32); whereas the contribution to the dispersion in the vertical plane (z) is given by the $\mathrm{w}_{1}$ term.

A global parameter is the momentum compaction factor

$\alpha_{\mathrm{p}}=\left(1 / 2 \pi \mathrm{R}_{\mathrm{cs}}{ }^{2}\right) \int \eta(\mathrm{s}) \mathrm{ds}$

Neglecting higher order terms in $\mathrm{L}_{\mathrm{s}} / \mathrm{R}$, we have

$\alpha_{\mathrm{p}}=\left(\mathrm{L}_{\mathrm{s}}^{2} \mathrm{w}_{0} / \pi^{2} \mathrm{R}\right)\left[\left(2 / \gamma_{\mathrm{s}}^{2} \underline{\mathrm{R}}_{\mathrm{s}}\right)+\left(1 / \mathrm{r}_{\mathrm{Ls}}\right)-<\chi_{\mathrm{w}}>\beta_{\mathrm{s}}{ }^{2} / \underline{\mathrm{R}}_{\mathrm{s}}\right]$

From this we derive the transition energy (in unit of rest energy)

$\gamma_{\mathrm{T}}=1 / \alpha_{\mathrm{p}}{ }^{1 / 2}$

In the case of no external bending field $\mathrm{B}_{\mathrm{ext}}=0,1 / \mathrm{r}_{\mathrm{Ls}}=0, \underline{\mathrm{R}}_{\mathrm{s}}=\mathrm{R}$,

$\gamma_{\mathrm{T}}=0.54\left(\gamma_{\mathrm{s}} \mathrm{R} / \mathrm{L}_{\mathrm{s}}\right) /\left(1-<\chi_{\mathrm{w}}>\beta_{\mathrm{s}}^{2} \gamma_{\mathrm{s}}^{2} / 2\right)$

In the other extreme case when the bending field is large enough so that $r_{L s}=R$ and $1 / \underline{\mathrm{R}}_{\mathrm{S}}=0$,

$\gamma_{\mathrm{T}} \quad=0.767 \mathrm{R} / \mathrm{L}_{\mathrm{s}}$

independent of the beam energy. In both cases, as long as $\mathrm{R}>>\mathrm{L}_{\mathrm{s}}$, for low energy particle beams $\gamma_{\mathrm{s}}<<\gamma_{\mathrm{T}}$, and the beam motion is always considerably well below the transition energy. In absence of an external field this is always true, no matter what is the beam energy. 


\section{Natural Chromaticity}

We have seen that the length of a period varies with the particle momentum according to $\mathrm{L}=\mathrm{L}_{\mathrm{s}}\left(1+\delta / \gamma_{\mathrm{s}}{ }^{2}\right)$. On the other end, the phase advance per period depends only on the RFQ parameter B and not on the period length. B varies inversely to $\gamma$, so that the dependence on the momentum error of the phase advance per period is

$$
\mu \quad=\quad \mu_{\mathrm{s}}\left(1-\beta_{\mathrm{s}}^{2} \chi_{\mu} \delta\right)
$$

where

$$
\chi_{\mu}=\left(\mathrm{B}_{\mathrm{s}} / v_{\mathrm{s}}\right) \partial v / \partial \mathrm{B}
$$

For a period with a phase advance close to $90^{\circ}, \chi_{\mu}=1.0522$. The number of periods depends on the momentum error as $n=n_{s} /\left(1-\delta / \gamma_{s}^{2}\right)$, which is a result quite different from that of a conventional storage ring where the periodicity is actually independent of the momentum error. As a consequence the total betatron tune,

$$
v=v_{\mathrm{s}}\left\{1+\left[1-\beta_{\mathrm{s}}{ }^{2}\left(1+\chi_{\mu}\right)\right] \delta\right\},
$$

from which we can estimate the natural chromaticity

$$
\xi=\left(1 / v_{\mathrm{s}}\right) \mathrm{d} v / \mathrm{d} \delta=1-\beta_{\mathrm{s}}{ }^{2}\left(1+\chi_{\mu}\right),
$$

different from that of conventional storage rings.

Another chromatic property is the variation of the amplitude lattice function $\beta_{\mathrm{L}}$ with the momentum error because it is directly proportional to the period length and depends on the RFQ parameter B.

$$
\beta_{\mathrm{L}} / \mathrm{L}=\left(\beta_{\mathrm{Ls}} / \mathrm{L}_{\mathrm{s}}\right)\left\{1-\left[1-\beta_{\mathrm{s}}^{2}\left(1-\chi_{\beta}\right)\right] \delta\right\}
$$

where

$$
\chi_{\beta}=\left(\mathrm{B}_{\mathrm{s}} \mathrm{L}_{\mathrm{s}} / \beta_{\mathrm{LS}}\right) \partial\left(\beta_{\mathrm{L}} / \mathrm{L}\right) / \partial \mathrm{B}
$$

Again, for a phase advance of $90^{\circ}$ per period, and for the maximum value of $\beta_{\mathrm{L}}$, $\chi_{\beta}=-0.593$.

The same considerations apply to the Twiss parameter that measures the rotation of the beam ellipse $\alpha_{L}$ in the phase space. This does not depend with the period length but varies with the RFQ parameter B, so that

$\alpha_{\mathrm{L}}=\alpha_{\mathrm{LS}}\left(1-\beta_{\mathrm{s}}^{2} \chi_{\alpha} \delta\right)$ 
where $\chi_{\alpha}=0.151$ for the same $90^{\circ}$ phase advance per period.

\section{Periodicity}

There is the focusing periodicity with period $\mathrm{L}$ and there is the geometrical periodicity, that is the CRFQ circumference $2 \pi R$. The lattice parameters $\beta_{\mathrm{L}}$ and $\alpha_{\mathrm{L}}$, the orbit distortion $\underline{u}$ and the dispersion $\eta$ have the periodicity of the focusing $L$, but do not follow necessarily the periodicity of the circumference. This is a very important point differentiating the CRFQ from the conventional storage ring. In the latter, the presence of magnets imposes that the lattice functions and dispersion are periodic functions of both the focusing period and of the circumference, because the latter is a multiple of the former. This is not the case in the CRFQ, since the number $n_{s}$ of periods per revolution is not necessarily an integer. This fact is expected to improve considerably the stability of motion versus manufacturing imperfections that, moreover, are also of different nature than those in magnetic conventional storage rings.

The beam is made of particles with a spread of momentum values. Thus, as we have seen previously, the periodicity $n=n_{s} /\left(1+\delta / \gamma_{s}^{2}\right)$ and varies with $\delta$. It is, therefore, important that the momentum spread is sufficiently small to avoid that part of the beam sits across an integral value of $\mathrm{n}$ that is $\delta<\gamma_{\mathrm{s}}^{2} / 4 \mathrm{n}_{\mathrm{s}}$.

\section{Initial Conditions}

The equations of motion Eq.s (23 and 24) have been derived for a particle that is at the unspecified location $s=0$ along the circumference at the time $t=0$ when the RF voltage reaches the maximum. At that location the amplitude lattice function $\beta_{\mathrm{L}}=\beta_{\max }$ and $\alpha_{\mathrm{L}}=0$. Also, at the same location and instant, the orbit distortion and dispersion have the maximum value. Differently from the conventional storage ring with magnets, where the lattice and dispersion functions have a locally fixed configuration, in the CRFQ the same quantities are not fixed but travel with the particle. Since particles reach the arbitrary chosen location $s=0$ at different times when the RF voltage has also different values, the lattice functions vary from one particle to the other according to their time lag. On the other hand, at a given time, say $t=0$, the RF voltage distribution is constant around the ring and, in particular, it may acquire the maximum value. Thus, at the same time $\mathrm{t}=0$, all particles in the beam experience the same maximum value of the lattice and dispersion functions. It derives that, for a continuous and uniform circulating beam, after several revolutions, as long as the periodicity $n_{s}$ is not an integer, the cross-section at any location is circular with a radius corresponding to the large value of $\beta_{\mathrm{L}}$. At the same time the divergence is also constant along the circumference and given by the inverse of $\beta_{\mathrm{L}}$.

\section{Transverse Space Charge Limitations}

Let a be the average radius of the cross-section, $\mathrm{N}$ the total number of ions in the beam, and introduce a bunching factor $f_{b}$ defined as the ratio of beam peak current to average current. Assuming also uniform transverse distribution, the electric field from space charge has the following components 
$\mathrm{E}_{\mathrm{x}}=\mathrm{Q}$ e $\mathrm{Nf}_{\mathrm{b}} \mathrm{x} / \pi \mathrm{R}^{2}$

$\mathrm{E}_{\mathrm{z}}=\mathrm{Q}$ e N f $\mathrm{z} / \pi \mathrm{R} \mathrm{a}^{2}$

After introducing the space-charge fields the equations of motion Eq.s (21 and 22) are modified as follows

$\mathrm{d}^{2} \mathrm{u} / \mathrm{ds}^{2}-\left(\mathrm{B}_{0} / \mathrm{L}^{2}\right) \mathrm{u} \cos (\mathrm{ks})-\left(\mathrm{K} / \mathrm{a}^{2}\right) \mathrm{u}=1 / \underline{\mathrm{R}}$

$\mathrm{d}^{2} \mathrm{v} / \mathrm{d} \mathrm{s}^{2}+\left(\mathrm{B}_{0} / \mathrm{L}^{2}\right) \mathrm{v} \cos (\mathrm{ks})-\left(\mathrm{K} / \mathrm{a}^{2}\right) \mathrm{v}=1 / \underline{\mathrm{R}}$

where

$\mathrm{K}=\mathrm{Q}^{2} \mathrm{e}^{2} \mathrm{Nf}_{\mathrm{b}} / \pi \mathrm{RAm} \gamma \beta^{2} \mathrm{c}^{2}$

The space charge term is a constant along the circumference of the CRFQ and does not oscillate periodically like the restoring force. Both the restoring force and the spacecharge force have the same momentum dependence since both vary like $1 / \gamma \beta^{2}$. The space charge effect is to reduce the focusing by the same amount in both planes. The phase advance per period will decrease by an amount depending on the constant K. Like in the straight RFQ [4] it is expected that a space-charge limit be encountered when the phase advance per period is lowered, for instance, from $90^{\circ}$ down to $60^{\circ}$ or $45^{\circ}$. At the same time also the lattice functions, orbit distortion and dispersion are modified by amounts that depend on the strength $\mathrm{K}$ of the space charge forces.

The space-charge limit corresponding to a phase advance depression from $90^{\circ}$ down to $45^{\circ}$ is

$\Delta=\mathrm{K}_{\mathrm{s}} \mathrm{L}_{\mathrm{s}}^{2} / \pi^{2} \mathrm{a}^{2}=0.044$

At the same time the amplitude lattice function $\beta_{\text {Ls }}$ and the Twiss parameter $\alpha_{\text {Ls }}$ closely double their original values. For instance, $\beta_{\mathrm{Ls}}$ varies now between the minimum value $1.84 \mathrm{~L}_{\mathrm{s}}$ and the maximum value $3.69 \mathrm{~L}_{\mathrm{s}}$, whereas the maximum value of $\alpha_{\mathrm{Ls}}$ is \pm 2.88 . The orbit distortion and dispersion are still given by Eqs. (30 and 39), except that the universal function $\mathrm{w}(\mathrm{s})$ now oscillates between $\mathrm{w}_{\min }=55.72$ and $\mathrm{w}_{\max }=78.60$. Thus, once the space charge limit of Eq. (57) is reached, the beam cross-section has increased by at most $40 \%$.

The space-charge limit as defined here for the CRFQ and expressed by Eq. (57) is of quite different nature than that usually encountered in a conventional storage ring with magnets. In the latter case the maximum beam current that can be stored is given by a small fraction of change of the betatron tune, that is of the total number of betatron oscillations per turn. The limit given by Eq. (57) is considerably higher and yields a

\footnotetext{
* Actually in principle it should be possible (in absence of errors) to lower the phase advance per period even down to zero. It is speculated [7] that at that level an organized formation of particles, called "crystalline beams" may appear with the help of a sufficiently fast cooling technique, like laser cooling.
} 
reduction of the betatron tune by a factor of 2 . The difference is caused by the different nature of the errors in the two rings and by the fact that in the CRFQ the ring circumference is not in an integral relation with the focusing periodicity, as it is always the case in the conventional storage ring.

\section{Racetrack-shaped RFQ}

We have described a Circular RFQ storage ring and have investigated the motion of particles. It is sometime more useful to consider a storage ring based on the same RFQ principle of focusing but of different shape. For instance the shape of a racetrack as shown in Figure 6 would also allow long straight sections to accommodate several functions and equipment. The racetrack has a geometrical periodicity of 2 , with two arcs of radius $\mathrm{R}$ and two straight sections each of length $\mathrm{H}$. The equations of motion are still given by Eq.s (23 and 24) where the curvature term at the r. h. side is now a periodic function with period $(\pi \mathrm{R}+\mathrm{H})$. In the straight sections the $r$. h. side vanishes identically since, we assume, there is no external bending field. We continue assuming that $\mathrm{R}$ and $\mathrm{H}$ are much larger than the focusing periodicity $L=\beta \lambda$. The "free betatron" solution is unchanged and as described for the CRFQ, but the orbit distortion and dispersion have now a different behavior that we shall examine below. For simplicity we shall concentrate only on the solution of Eq. (23). Also we shall study only the case where there is no external field, or the case where the curvature $1 / \mathrm{R}$ is only partially compensated. In the case $1 / \underline{R}=0$ the orbit distortion $\underline{\mathrm{u}}$ is always null all around the racetrack.

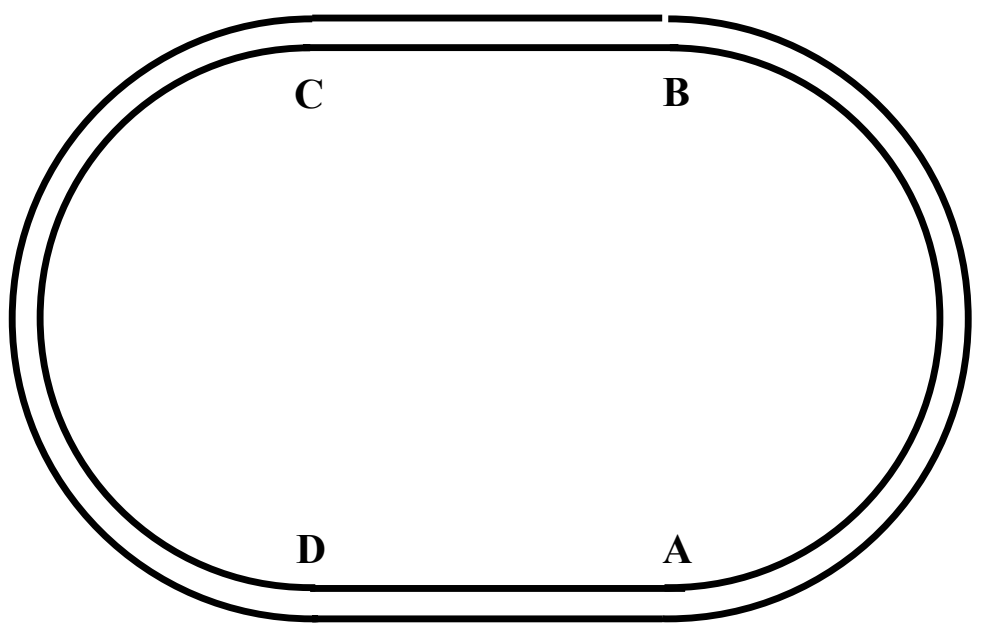

Figure 6. The Racetrack-shaped RFQ Storage Ring

Consider a particle that starts its motion at the location $\mathbf{A}$, at the beginning of the arc $\mathbf{A B}$. Its motion can be described by a vector $U(s)$ with components $u(s)$ and $u^{\prime}(s)=d u(s) / d s$. The initial values, at the location $\mathbf{A}$, are $\mathrm{U}_{0}=\left(\mathrm{u}_{0}, \mathrm{u}^{\prime}{ }_{0}\right)$. The equations of motion in the arc $\mathbf{A B}$ are, in vector notation, 
$\mathrm{U}(\mathrm{s})=A_{1}(\mathrm{~s}) \mathrm{C}+\underline{\mathrm{U}}(\mathrm{s})$

where $\mathrm{C}=\left(\mathrm{c}_{1}, \mathrm{c}_{2}\right)$ is a vector of two constants, and $A_{1}(\mathrm{~s})$ is the transfer matrix from the starting point $\mathbf{A}$ (defined as s $=0$ ) to any other point in the arc at the arc distance s. The first term at the r.h. side of Eq. (58) is the "free betatron" oscillation around the orbit distortion, described by the vector $\underline{\mathrm{U}}(\mathrm{s})$ of components $\underline{\mathrm{u}}(\mathrm{s})$ and $\underline{\mathrm{u}}^{\prime}(\mathrm{s})$. Obviously $\mathrm{C}=\mathrm{U}_{0}-\underline{\mathrm{U}}_{0}$, with $\underline{\mathrm{U}}_{0}$ the initial value of the orbit distortion. At the location $\mathbf{B}$, at the end of the $\operatorname{arc} \mathbf{A} \mathbf{B}$, of length $\pi R$,

$\mathrm{U}_{\mathrm{B}}=A_{1}(\mathrm{~s}=\pi \mathrm{R})\left(\mathrm{U}_{0}-\underline{\mathrm{U}}_{0}\right)+\underline{\mathrm{U}}_{1 \mathbf{B}}$

where $\underline{\mathrm{U}}_{1 \mathbf{B}}=\underline{\mathrm{U}}(\mathrm{s}=\pi \mathrm{R})$ is the orbit distortion at the end of the arc. A mismatch is encountered at the location $\mathbf{B}$ when the particle leaves the arc with curvature 1/R and enters the straight section of length $\mathrm{H}$ and zero curvature. Carrying the particle through the end of the drift, to the location $\mathbf{C}$, for the completion of one geometrical period of length $\mathrm{H}+\pi \mathrm{R}$,

$\mathrm{U}_{1}=B_{1} A_{1}\left(\mathrm{U}_{0}-\underline{\mathrm{U}}_{0}\right)+B_{1} \underline{\mathrm{U}}_{1 \mathbf{B}}$,

where $A_{1}$ is the transfer matrix along the arc $\mathbf{A B}$ of the first geometrical period, and $B_{1}$ the one for the following drift between $\mathbf{B}$ and $\mathbf{C}$. In the following we shall denote with $A_{\mathrm{m}}$ and $B_{\mathrm{m}}$ the transfer matrices corresponding to the m-th geometrical period, and, more compactly, $\mathrm{M}_{\mathrm{m}}=B_{\mathrm{m}} A_{\mathrm{m}}$. The second term at the r. h. side of Eq. (60) is a new oscillation induced by the mismatch of the curvature at the location B. Every time the particle leaves an arc and enters a drift, and vice versa, a new oscillation is induced. After iterating, at the end of the $\mathrm{n}$-th geometrical period, that is $\mathrm{n} / 2$ revolution,

$\mathrm{U}_{\mathrm{n}}=\mathrm{M}_{\mathrm{n}}\left(\mathrm{U}_{\mathrm{n}-1}-\underline{\mathrm{U}}_{\mathrm{n}-1}\right)+B_{\mathrm{n}} \underline{\mathrm{U}}_{\mathrm{n} \mathbf{B}}$

$\mathrm{U}_{\mathrm{n}}=\mathrm{M}_{1}^{\mathrm{n}}\left(\mathrm{U}_{0}-\underline{\mathrm{U}}_{0}\right)+B_{\mathrm{n}} \underline{\mathrm{U}}_{\mathrm{n} \mathbf{B}}+\sum_{\mathrm{m}=2, \mathrm{n}} \mathrm{M}_{\mathrm{m}}^{\mathrm{n}}\left(B_{\mathrm{m}-1} \underline{\mathrm{U}}_{\mathrm{m}-1, \mathbf{B}}-\underline{\mathrm{U}}_{\mathrm{m}-1}\right)$

where $\mathrm{M}_{\mathrm{m}}{ }^{\mathrm{n}}$ is a chain product of transfer matrices

$M_{m}{ }^{n}=M_{n} M_{n-1} M_{n-2} \ldots M_{m+2} M_{m+1} M_{m}$

valid for $\mathrm{m}<\mathrm{n}$, and $\mathrm{M}_{\mathrm{n}}{ }^{\mathrm{n}}=\mathrm{M}_{\mathrm{n}}$. Each transfer matrix $\mathrm{M}_{\mathrm{m}}$ is made of a phase advance per geometric period $\mu / 2$ that rotates the vector $U_{\mathrm{m}-1}$ by an angle of the same amount, and an ellipse dilation, measured by the lattice functions $\beta_{\mathrm{L}}$ and $\alpha_{\mathrm{L}}$, which varies from one geometric period to the next. It is convenient to decompose the transfer matrix

$\mathrm{M}_{\mathrm{m}}=R_{\mathrm{m}}^{-1} S(\mu / 2) R_{\mathrm{m}-1}$

where $S(\mu / 2)$ is a rotation by the angle $\mu / 2$. In general 
$S(\alpha)=\left(\begin{array}{cc}\cos \alpha & \sin \alpha \\ -\sin \alpha & \cos \alpha\end{array}\right)$.

The dilation matrix, defined at the end of the m-th geometric period,

$R_{\mathrm{m}}=\left(\begin{array}{cc}1 / \sqrt{ } \beta_{\mathrm{m}} & 0 \\ \alpha_{\mathrm{m}} / \sqrt{ } \beta_{\mathrm{m}} & \sqrt{ } \beta_{\mathrm{m}}\end{array}\right)$.

Thus

$\mathrm{M}_{\mathrm{m}}{ }^{\mathrm{n}}=R_{\mathrm{n}}{ }^{-1} S[(\mathrm{n}-\mathrm{m}+1) \mu / 2] R_{\mathrm{m}-1}$

and

$\mathrm{M}_{\mathrm{m}}{ }^{\mathrm{n}} B_{\mathrm{m}-1}=R_{\mathrm{n}}{ }^{-1} S\left[(\mathrm{n}-\mathrm{m}+1) \mu / 2+\mu_{\mathrm{L}}\right] R_{\mathrm{m}-1, \mathrm{~B}}$

with $\mu_{\mathrm{L}}$ the phase advance per straight section. Let us operate the coordinate transformation $\mathrm{E}_{\mathrm{m}}=R_{\mathrm{m}} \mathrm{U}_{\mathrm{m}}$ that transforms ellipses to circles. Combining all together, Eq. (62) finally becomes

$$
\begin{aligned}
\mathrm{E}_{\mathrm{n}}= & S(\mathrm{n} \mu / 2)\left(\mathrm{E}_{0}-\underline{\mathrm{E}}_{0}\right)+ \\
& \sum_{\mathrm{m}=2, \mathrm{n}+1} S\left[(\mathrm{n}-\mathrm{m}+1) \mu / 2+\mu_{\mathrm{L}}\right] \underline{\mathrm{E}}_{\mathrm{m}-1, \mathrm{~B}}-\sum_{\mathrm{m}=2, \mathrm{n}} S[(\mathrm{n}-\mathrm{m}+1) \mu / 2] \underline{\mathrm{E}}_{\mathrm{m}-1}
\end{aligned}
$$

This is as far as we can go. The first term at the r. h. side of Eq. (69) represents the usual propagation of the "free betatron" oscillation. The second term is the orbit distortion caused by the mismatch in curvature when the particles leaves an arc and enter the subsequent drift. The last term is the same, except that the mismatch is between the drift and the following arc. It is easily seen that, in the limit $\mathrm{H}=0$, also $\mu_{\mathrm{L}}=0, \underline{\mathrm{E}}_{\mathrm{mB}}=\underline{\mathrm{E}}_{\mathrm{m}}$ and the two orbit distortion terms cancel each other, leaving only the local orbit distortion $\underline{E}_{n}$, recovering thus the result of the CRFQ.

In view of the transformation from elliptical to circular variables, the orbit distortion vectors $\underline{E}_{m}$ and $\underline{E}_{m B}$ can be treated essentially as constants and taken out of the summations at the r. h. side of Eq. (69). Also, we have

$\sum_{\mathrm{m}=2, \mathrm{n}+1} S\left[(\mathrm{n}-\mathrm{m}+1) \mu / 2+\mu_{\mathrm{L}}\right]=S\left(\mu_{\mathrm{L}}\right) \sum_{\mathrm{m}=2, \mathrm{n}} S[(\mathrm{n}-\mathrm{m}+1) \mu / 2]+S\left(\mu_{\mathrm{L}}\right)$

and, using complex notation,

$$
\begin{aligned}
& \{1-\exp [\mathrm{i}(\mathrm{n}-1) \mu / 2]\}[\exp (\mathrm{i} \mu / 2)-1] \\
& \sum_{\mathrm{m}=2, \mathrm{n}} S[(\mathrm{n}-\mathrm{m}+1) \mu / 2] \quad=\quad-2(1-\cos \mu / 2)
\end{aligned}
$$


Thus, an instability appears when the phase advance per revolution $\mu=4 \pi q$ and $q$ is an integer. In terms of betatron tune, this corresponds to the first order resonance $v=2 q$. This result was expected: at the resonance, the orbit distortion would grow indefinitely and cause the loss of the beam. Because the racetrack storage ring has a geometric periodicity of 2 , betatron tunes with even integer values are to be avoided. For instance, one can adopt the range

$2 q+1 / 2<v<2 q+3 / 2$

in which case the orbit distortion will grow no more than a factor 2 of the original value.

This analysis can be easily extended to the case of a number of insertions larger than 2 . Increasing the number of geometrical periods is expected to improve the stability of motion, so that one can relax on the stability condition of Eq. (72).

\section{Rods with Convolution}

To allow acceleration in the CRFQ we need a longitudinal field along the main axis of motion. Shaping the four rods so that they show convolution on the sides opposite to each other as shown in Figure 7 can create this.

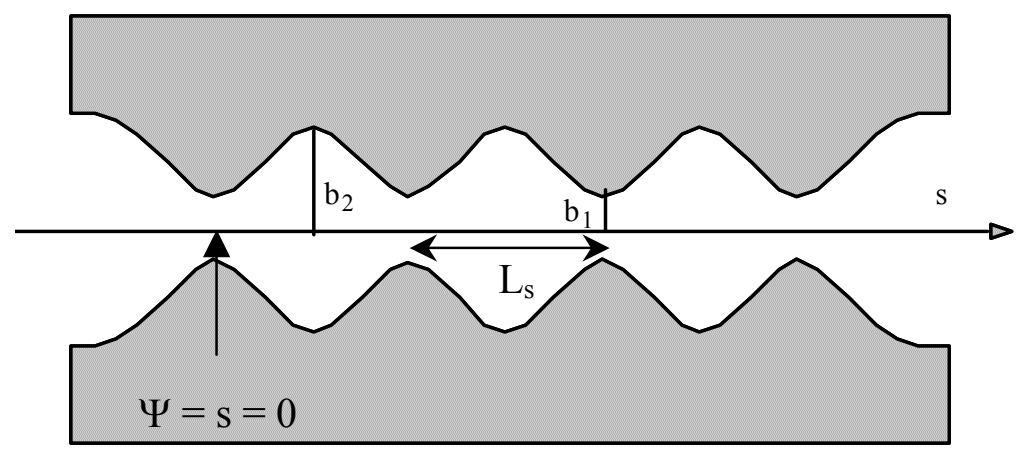

Figure 7. Coils shaped with convolution to provide for acceleration.

As in the conventional linear RFQ [4], the period of the convolution equals that of the focusing $L_{s}=\beta_{s} \lambda$. We shall denote with $b_{1}$ the inner minor radius, and with $b_{2}$ the major inner radius. Since the ring has to close mechanically on itself, it is now required that the number of periods $n_{s}=2 \pi R / L_{s}$ is exactly an integer, with the consequences we have already discussed.

The addition of the coil convolution modifies the field potentials in the way discussed in details in Appendix C. Retaining only the linear terms, and in the approximation that $\mathrm{L}>>$ b, it can be shown that the equations for the transverse motion, Eq.s (23 and 24), are essentially unchanged. A longitudinal electric field appears on axis $(\rho=0)$

$\mathrm{E}_{\psi} \quad=\quad \mathrm{V}_{0} \xi \mathrm{k}_{\mathrm{s}} \sin \left(\mathrm{k}_{\mathrm{s}} \mathrm{s}\right) \cos (\omega \mathrm{t})$ 
where the factor $\xi$ is given in Appendix C by Eq. (C14). The longitudinal motion of the particle is thus no longer a drift, but it is described by energy oscillations. Let $E$ be the total energy of any given particle; the energy gain per unit of path length is

$\mathrm{d} E / \mathrm{ds} \quad=\quad \mathrm{Qe} \mathrm{V}_{0} \xi \mathrm{k}_{\mathrm{s}} \sin \left(\mathrm{n}_{\mathrm{s}} \psi\right) \cos (\omega \mathrm{t})$

Introduce the RF phase angle

$\phi \quad=\quad \mathrm{n}_{\mathrm{s}} \psi-\omega \mathrm{t}$

After neglecting a fast oscillating term, Eq. (74) becomes

$\mathrm{d} E / \mathrm{d} \mathrm{s} \quad=\quad(1 / 2) \mathrm{Qe} \mathrm{V}_{0} \xi \mathrm{k}_{\mathrm{s}} \sin \phi$

At the same time the time derivative of both sides of Eq. (75) gives

$\mathrm{d} \phi / \mathrm{dt}=\mathrm{n}_{\mathrm{s}} \omega_{\mathrm{o}}(\mathrm{E})-\omega$

where $\omega_{0}(E)=d \psi / d t$ is the angular revolution frequency which varies with the beam energy.

By definition, the reference particle, that has momentum $\mathrm{p}_{\mathrm{s}}$ and total energy $E_{\mathrm{s}}$, has the angular revolution frequency $\omega_{\mathrm{os}}$ satisfying the synchronous condition $\mathrm{n}_{\mathrm{s}} \omega_{\mathrm{os}}=\omega$. The reference particle is thus also called the synchronous particle. The RF phase angle $\phi_{\mathrm{s}}$ of this particle is constant, and the energy equation is

$\mathrm{d} E_{\mathrm{s}} / \mathrm{ds}=(1 / 2) \mathrm{Qe} \mathrm{V}_{0} \xi \mathrm{k}_{\mathrm{s}} \sin \phi_{\mathrm{s}}$

It is convenient to take as canonical variables the energy difference $\mathrm{w}=E-E_{\mathrm{s}}$, and the $\mathrm{rf}$ phase angle $\phi$, and use the path length s along the reference distorted orbit as the independent variable. With a linear expansion around the synchronous particle

$\omega_{\mathrm{o}}(\mathrm{E}) \quad=\quad \omega_{\mathrm{os}}+\omega_{\mathrm{os}}\left(\eta_{\mathrm{s}} / \beta_{\mathrm{s}}^{2} E_{\mathrm{s}}\right) \mathrm{w}$.

where

$\eta_{\mathrm{s}} \quad=\gamma_{\mathrm{s}}^{-2}-\gamma_{\mathrm{T}}^{-2}$

and $\gamma_{\mathrm{T}}$ is the transition energy defined by Eqs. (42-44). We derive the equations of motion for any particle expanded around the synchronous conditions

$\begin{array}{lll}\mathrm{dw} / \mathrm{ds} & = & (1 / 2) \mathrm{Qe} \mathrm{V}_{0} \xi \mathrm{k}_{\mathrm{s}}\left(\sin \phi-\sin \phi_{\mathrm{s}}\right) \\ \mathrm{d} \phi / \mathrm{ds} & = & \left(\mathrm{k}_{\mathrm{s}} \eta_{\mathrm{s}} / \mathrm{Amc}^{2} \gamma_{\mathrm{s}} \beta_{\mathrm{s}}^{2}\right) \mathrm{w}\end{array}$ 
These equations are similar to those describing synchrotron oscillations in conventional particle accelerators. One can then use the same approach to estimate particle trajectories and the stability region in the phase space (RF bucket area and height). In particular, we can linearize the motion around the synchronous conditions to derive the equations for the energy oscillations

$\mathrm{d}^{2} \mathrm{w} / \mathrm{dt}^{2}+\Omega_{\mathrm{s}}^{2} \mathrm{~W}=0$

where the angular oscillation frequency

$\Omega_{\mathrm{s}}^{2}=-\mathrm{QeV}_{0} \xi \mathrm{k}_{\mathrm{s}}^{2} \eta_{\mathrm{s}} \cos \phi_{\mathrm{s}} / 2 \mathrm{Am} \gamma_{\mathrm{s}}$

In order for the motion to be stable it is required that $\eta_{\mathrm{s}} \cos \phi_{\mathrm{s}}<0$.

\section{Longitudinal Space-Charge Limitations for Bunched Beams}

In case of acceleration in the CRFQ the beam is bunched. Because of the very large frequency the beam bunches have a very small length that may be comparable to the transverse size. Each bunch is made of $\mathrm{N}_{\mathrm{b}}=\mathrm{N} / \mathrm{n}_{\mathrm{s}}$ particles, and has typically the shape of an ellipsoid of full length $2 \ell$ and cross-section radius a. We shall assume uniform charge distribution within the ellipsoid, that yields an electric field that has components exactly linear with the displacement of the test particle ( $\mathrm{x}, \mathrm{z}$ and $\mathrm{s}$ ) from the center of the bunch. The three field components are

$$
\begin{aligned}
& \mathrm{E}_{\mathrm{x}, \mathrm{z}}=3 \mathrm{QeN}_{\mathrm{b}}[1-\mathrm{f}(\ell / \mathrm{a})](\mathrm{x} \text { or } \mathrm{z}) / 2 \mathrm{a}^{2} \ell \\
& \mathrm{E}_{\mathrm{s}}=3 \mathrm{Qe} \mathrm{N}_{\mathrm{b}} \mathrm{f}(\ell / \mathrm{a}) \mathrm{s} / \mathrm{a}^{2} \ell
\end{aligned}
$$

where [8]

$$
\begin{aligned}
\mathrm{f}(\mathrm{p}) & =\left[\left(1-\mathrm{p}^{2}\right)^{1 / 2}-\mathrm{p} \arccos (\mathrm{p})\right] /\left(1-\mathrm{p}^{2}\right)^{3 / 2} & \text { for } \mathrm{p}<1 \\
& =1 / 3 & \text { for } \mathrm{p}=1 \\
& =\quad\left[\mathrm{p} \operatorname{arccosh}(\mathrm{p})-\left(\mathrm{p}^{2}-1\right)^{1 / 2}\right] /\left(\mathrm{p}^{2}-1\right)^{3 / 2} & \text { for } \mathrm{p}>1
\end{aligned}
$$

In the limit of long bunches, $\ell / \mathrm{a}->\infty$,

$$
\mathrm{f}(\ell / \mathrm{a}) \sim\left(\mathrm{a}^{2} / \ell\right) \ln (\ell / \mathrm{a})
$$

The equations of motion Eqs. (54 and 55) still apply with the exception that now, instead of Eq. (56), we have

$$
\mathrm{K}=3 \mathrm{Q}^{2} \mathrm{e}^{2} \mathrm{~N}_{\mathrm{b}}(1-\mathrm{f}) / 2 \ell \mathrm{Am} \gamma \beta^{2} \mathrm{c}^{2}
$$

All the considerations made earlier for the debunched beam still apply. In particular, the space-charge transverse limit given by Eq. (57) still holds. 
Similarly, the equation for the small-amplitude longitudinal oscillations, Eq. (83), is modified with introducing a term due to space charge as follows

$\mathrm{d}^{2} \mathrm{w} / \mathrm{dt}^{2}+\left(\Omega_{\mathrm{s}}^{2}-\Delta_{\mathrm{sc}}\right) \mathrm{w}=0$

where

$\Delta_{\mathrm{sc}} \quad=\quad 3 \mathrm{~N}_{\mathrm{b}} \mathrm{Q}^{2} \mathrm{e}^{2} \mathrm{f} / \mathrm{Am} \ell \mathrm{a}^{2} \beta_{\mathrm{s}}{ }^{2} \gamma_{\mathrm{s}}^{3}$

The longitudinal motion is stable as long as*

$\Omega_{\mathrm{s}}^{2}>\Delta_{\mathrm{sc}}$

\section{Applications of the CRFQ Storage Ring and Accelerator}

There are several applications we foresee for the CRFQ. We shall list below six of these applications; but, presumably, many more are possible. For each of the six applications we shall give an example in the following sections.

1. First of all, we are interested in a prototype for the demonstration of the principles of operations of the CRFQ. We need to demonstrate that indeed it is possible to achieve, through the very strong focusing, small beam dimensions. Also that it is possible to reach a space-charge limit considerably higher than that of conventional storage rings. The case of the storage of a $100-\mathrm{keV}$ proton beam is discussed. The four coils have no convolution since here there is no need to provide acceleration. The ring is of modest dimensions, with a radius $\mathrm{R}=0.5 \mathrm{~m}$. No external bending field is required since the bending of the particle trajectory is accomplished entirely with the help of the RF forces.

2. The next application is the storage of light and heavy ions (including protons) at low non-relativistic energies (for instance few $\mathrm{MeV} / \mathrm{u}$ ) for studies in Molecular and Atomic Physics. Acceleration is not required here. An external bending field may be necessary to control the orbit distortion and keep it within the aperture between the coils. The stored beam can be made to collide with a laser or other photon beams, electron beams or, head-on with beams of other ion species. The use of jets or foil targets during repetitive passages can also be exploited for research. This storage ring can also be used with laser/electron cooling, and for the demonstration (eventually) of "crystalline beams" [7]. Two such rings can also be made to intersect with each other for head-on collision of counter-moving light ions, like protons and boron, for the exploration of production of nuclear fusion power [9].

\footnotetext{
* It is actually speculated [7] that, in the limit the longitudinal space-charge forces cancel exactly the oscillation external restoring force, the motion reduces to drifts again, and an ordered state is reached called "crystalline beams".
} 
3. Storage of 1.75 to $3.24 \mathrm{MeV}$ proton beam with an intensity of $10-100 \mathrm{~mA}$. External bending field in this case is required. The device can be used as a compact source of collimated gamma and neutrons rays. For this purpose, a carbon foil is inserted and crossed by the beam turn-after-turn. Protons, by impinging on the target, produce secondary beams. A possible application of this device is the screening of objects and inspection of their content. Protons suffer a systematic energy loss when crossing the target that needs to be compensated; a modest acceleration is thus required. At this purpose, the coils have a convolution.

4. It is possible to use the CRFQ to accelerate ions (protons) over a relatively low energy range where the particle velocity varies appreciably. As an example, a compact medical accelerator is investigated. By properly convoluting the coils, a proton beam is accelerated from 20 to $250 \mathrm{MeV}$ at the repetition rate of 60 beam pulses with an intensity of about $10^{9}$ protons per pulse. There is obviously need of an external bending field that in turn needs also to be ramped. This device is certainly useful for the demonstration of the principle of acceleration in the CRFQ.

5. We shall also consider acceleration of relativistic ions (and protons, and electrons) with velocity close to the speed of light $(\beta \sim 1)$. Because of the large beam energy the device has now a very large size. The external bending field needs to be ramped with the change of the particle momentum but, because the particle velocity does not change much, the RF frequency also does not vary and it is kept constant during the operation.

6. Finally, we shall study the application of the CRFQ as a compact synchrotron radiation source. The example we report below deals with a $800-\mathrm{MeV}$ beam for the production of UV radiation. Because of the special behavior of the lattice and dispersion functions in the CRFQ storage ring, we expect a high beam brilliance and collimation. The conventional synchrotron radiation integrals to estimate the beam dimension at equilibrium and the properties of the radiation are given. Since the beam energy is constant, the external bending field is also kept constant. Acceleration is required to compensate for the energy lost to radiation but, at the same time, the RF will be also kept constant during operation.

\section{The CRFQ Prototype}

The main parameters of the prototype are listed in Table 1 below. This device serves for the demonstration of operation principles of the CRFQ. There are two major goals: (1) the demonstration of strong focusing by which it is possible to obtain very small beam dimensions, and (2) the achievement of large beam intensities well above those that can be obtained in conventional magnet storage ring.

The ring is small, with only 1-meter diameter, and thus of easy and inexpensive construction. Since acceleration is not required, the metal coils are smooth with a cylindrical shape of 1-centimeter diameter. The internal radius of the structure 
$\mathrm{b}=1 \mathrm{~cm}$. The RF excitation proposed is $200 \mathrm{MHz}$, with a wavelength $\lambda=150 \mathrm{~cm}$. For the study and experimental demonstration we plan the storage of $100-\mathrm{keV}$ proton beams. At this energy $\beta=0.0146$, and the resulting focusing period is $L=\beta \lambda=2.2 \mathrm{~cm}$. Aiming to a phase advance per period of $90^{\circ}$, in the limit of zero beam current, the peak RF voltage required is $142 \mathrm{kV}$, which yields an electric field of $14.2 \mathrm{MV} / \mathrm{m}$, below the surface limit. The estimated orbit distortion is at most $2 \mathrm{~mm}$, well within the coil aperture, so that there is no need of an external bending field to help confining the beam. Also the radius of the beam cross-section a $\sim 1 \mathrm{~mm}$ fits well within the available aperture. All the other lattice parameters are given in Table 1.

Table 1. Parameters of the CRFQ Prototype

\begin{tabular}{|l|r|}
\hline Type of particles & Protons \\
\hline Charge State, $\mathrm{Q}$ & 1 \\
\hline Mass Number, A & 1 \\
\hline Rest Energy, mc ${ }^{2}$ & $938.26 \mathrm{MeV}$ \\
\hline Kinetic Energy & $100 \mathrm{keV}$ \\
\hline$\beta$ & 0.01460 \\
\hline$\gamma$ & 1.00011 \\
\hline Momentum, $\mathrm{p}$ & $13.70 \mathrm{MeV} / \mathrm{c}$ \\
\hline Magnetic Rigidity, B $\rho$ & $0.4570 \mathrm{kG}-\mathrm{m}$ \\
\hline Excitation RF & $200 \mathrm{MHz}$ \\
\hline Wavelength, $\lambda$ & $149.9 \mathrm{~cm}$ \\
\hline Focusing Period, $\mathrm{L}$ & $2.19 \mathrm{~cm}$ \\
\hline Internal Radius, $\mathrm{b}$ & $10 \mathrm{~mm}$ \\
\hline RF Peak Voltage, $\mathrm{V}_{\mathrm{o}}$ & $142.2 \mathrm{kV}$ \\
\hline Phase Adv. / Period & $90^{\circ}$ \\
\hline$\beta_{\mathrm{L}}$ - max & $4.11 \mathrm{~cm}$ \\
\hline Major Radius, $\mathrm{R}$ & $50 \mathrm{~cm}$ \\
\hline Max. Orbit Distortion & $1.92 \mathrm{~mm}$ \\
\hline Max. Dispersion & $0.383 \mathrm{~cm}$ \\
\hline No. of Periods, $\mathrm{n}_{\mathrm{s}}$ & 143.56 \\
\hline Betatron Tune, $v_{\mathrm{s}}$ & 35.89 \\
\hline Transition Energy, $\gamma_{\mathrm{T}}$ & 12.34 \\
\hline Chromaticity & 0.99956 \\
\hline Norm. Emittance & $0.30 \pi \mathrm{mm} \mathrm{mrad}$ \\
\hline Actual Emittance & $20.55 \pi \mathrm{mm} \mathrm{mrad}$ \\
\hline Beam Radius, a & $0.919 \mathrm{~mm}$ \\
\hline No. of Protons, N & $1.25 \times 10^{11}$ \\
\hline Revol. Frequency & $1.393 \mathrm{MHz}$ \\
\hline Beam Pulse Length & $0.54 \mu \mathrm{s}$ \\
\hline Beam Current & $37 \mathrm{~mA}$ \\
\hline
\end{tabular}


Adopting the space-charge limit given by Eqs. (56 and 57), with a bunching factor $\mathrm{f}_{\mathrm{b}}=1.33$, the expected intensity that can be stored is $\mathrm{N}=1.25 \times 10^{11}$, two orders of magnitude larger than that can be reached in a equivalent conventional magnetic storage ring.

Another function of the CRFQ prototype is learning how it is possible to inject a short beam pulse, how to power the device with RF, and how to provide instrumentation for the beam diagnostic. The experimental apparatus may look like the one shown schematically in Figures 8 and 9. An ion source sits on a platform at $35 \mathrm{kV}$ and provides a short proton pulse at relatively low duty cycle. The beam pulse is accelerated in a short linear RFQ to $100 \mathrm{keV}$ operating at the same frequency of $200 \mathrm{MHz}$. A short section joins the linear RFQ to the CRFQ providing matching and beam transfer. The CRFQ is encased in a tank with an opening at the bottom for the location of a vacuum pump. The four coils sit on a platform and are supported with blocks of G-10. A number of RF cavities excite the structure from the outside. Feed-through are provided on the top and bottom of the tank for connections to power supplies, beam diagnostics and RF sources. To perform the experiment is sufficient to run and store one beam pulse every few minutes for just few seconds. The low duty cycle will remove the need of water-cooling.

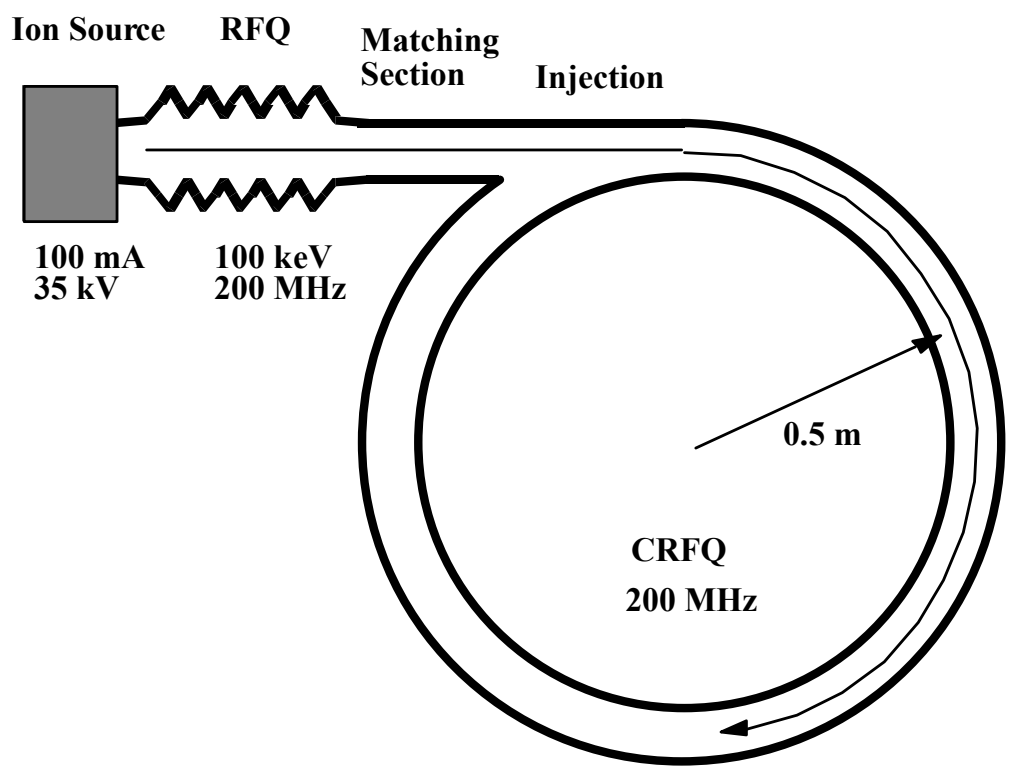

Figure 8. Experimental layout of the CRFQ prototype 


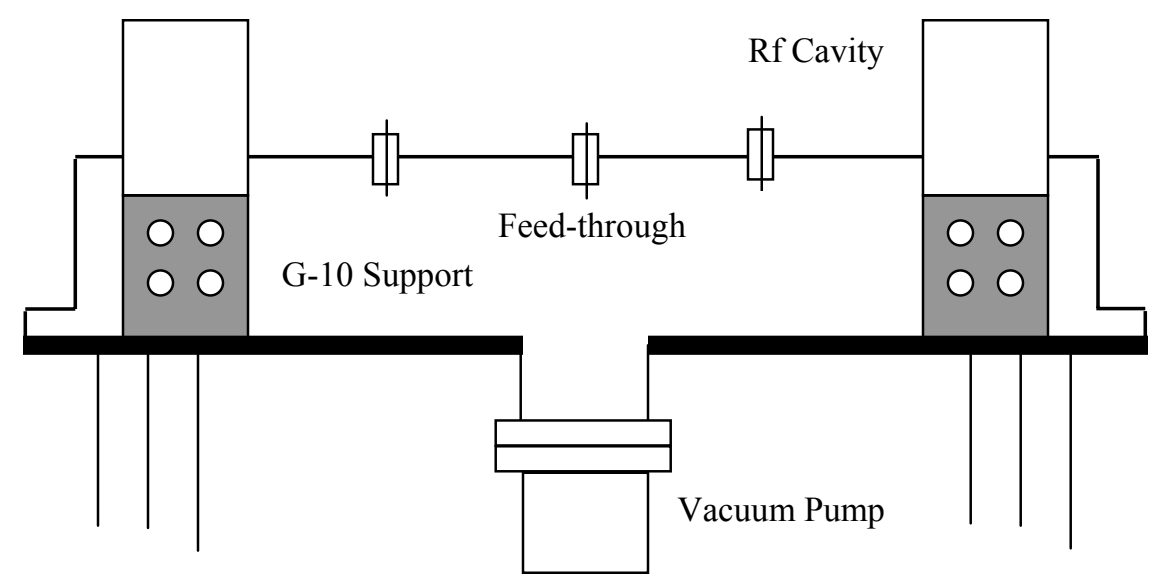

Figure 9. Encasement of the CRFQ Prototype

\section{CRFQ Ion Storage Rings}

There are several storage rings for protons and ions in the world. They usually operate at a relatively low energy and have the capability to store different ion species one at the time, of course. Thus, instead of the particle energy the maximum magnetic rigidity of the ring is quoted. The main requirement of these storage rings is a beam with very small momentum spread and high particle density. We believe, as the example below illustrates, that the CRFQ can be used as an ideal storage ring for ions. It allows considerably more compactness of device, and very dense and intense ion beams. We shall consider, as an example, a magnetic rigidity of $3 \mathrm{~T}-\mathrm{m}$. The main parameters of the storage ring are listed in Table 2 . Three species are considered: proton, ${ }^{9} \mathrm{Be}^{+}$and ${ }^{24} \mathrm{Mg}^{+}$. 
Table 2. Parameters of a CRFQ Storage Ring for Ions

\begin{tabular}{|l|r|r|r|}
\hline & \multicolumn{1}{|c|}{ Proton } & \multicolumn{1}{|c|}{${ }^{9} \mathrm{Be}^{+}$} & ${ }^{24} \mathrm{Mg}^{+}$ \\
\hline Charge State & 1 & 1 & 1 \\
\hline Mass Number & 1 & 9 & 24 \\
\hline Kin. Energy, MeV/u & 361.4 & 5.31 & 0.75 \\
\hline$\beta$ & 0.6920 & 0.1059 & 0.0399 \\
\hline$\gamma$ & 1.3852 & 1.0057 & 1.0008 \\
\hline Momentum, MeV/u/c & 899.4 & 99.9 & 37.5 \\
\hline Excitation RF, MHz & 40 & 40 & 40 \\
\hline Wavelength, $\lambda, \mathrm{cm}$ & 749.5 & 749.5 & 749.5 \\
\hline Focus. Period, $\mathrm{L}, \mathrm{cm}$ & 518.6 & 79.4 & 29.9 \\
\hline Int. Radius,, $\mathrm{cm}$ & 1.0 & 1.0 & 1.0 \\
\hline RF Voltage, $\mathrm{V}_{\mathrm{o}}, \mathrm{kV}$ & 7.9 & 51.5 & 136.6 \\
\hline Phase Adv. / Period & $90^{\circ}$ & $90^{\circ}$ & $90^{\circ}$ \\
\hline$\beta_{\mathrm{L}}-$ max, $\mathrm{m}$ & 9.74 & 1.49 & 0.56 \\
\hline Major Radius, $\mathrm{R}, \mathrm{cm}$ & 300 & 300 & 300 \\
\hline Max. Dispersion, $\mathrm{m}$ & 17.9 & 0.42 & 0.060 \\
\hline No. of periods, $\mathrm{n}_{\mathrm{s}}$ & 3.634 & 23.75 & 63.02 \\
\hline Betatron Tune, $v_{\mathrm{s}}$ & 0.909 & 5.937 & 15.755 \\
\hline Transition Energy, $\gamma_{\mathrm{T}}$ & 0.433 & 2.052 & 5.420 \\
\hline Chromaticity & 0.0173 & 0.9770 & 0.9967 \\
\hline Norm. Emit., $\pi \mathrm{mm} \mathrm{mrad}$ & 0.30 & 0.30 & 0.30 \\
\hline Act. Emit., $\pi \mathrm{mm} \mathrm{mrad}$ & 0.313 & 2.817 & 7.511 \\
\hline Beam Radius, a, mm & 1.75 & 2.05 & 2.05 \\
\hline No. of Ions, $\mathrm{x} 10^{12}$ & 0.109 & 1.35 & 3.60 \\
\hline Rev. Frequency, MHz & 11.06 & 1.684 & 0.635 \\
\hline Beam Pulse Length, $\mu \mathrm{s}$ & 0.068 & 0.445 & 1.182 \\
\hline Beam Current, A-part & 0.191 & 0.363 & 0.366 \\
\hline
\end{tabular}

The device is intended only as a storage ring and no acceleration is required. Thus, the coils have no convolution. In absence of an external bending field the orbit distortion is too large and would exceed the aperture between the coils, therefore, an external bending field is required. We take $B_{\text {ext }}=10 \mathrm{kG}$ giving a bending radius $\rho=3 \mathrm{~m}$. The bending field is constant, and is provided by an annular dipole magnet covering the entire circumference of the CRFQ, as shown in Figure 10. The magnet gap needs to be only few centimeters high. To cancel exactly the residual curvature, that is $1 / \underline{R}=0$, we adjust the radius of the CRFQ so that $R=\rho=3 \mathrm{~m}$. The radius a of the beam cross-section is no more than $2 \mathrm{~mm}$, in all cases, and thus fits the available aperture between the coils. We have taken an excitation RF of $40 \mathrm{MHz}$. The required peak RF voltage is reasonable. There are some major differences between the case of storage of protons to that of heavier ions. The number of periods is low in the case of protons and comparable to that of a conventional magnetic storage ring, but it is fractional and, thus, one would expect also for the protons a considerable more stability of motion, with less susceptibility to errors. The lattice and dispersion function are also comparable, and even larger, to that of conventional rings. This may suggest that a higher RF frequency could be chosen for the 
proton case. A larger space-charge limit is expected also for protons, but only a few factor larger than that compared of a conventional storage ring. In the case of heavier ions the number of periods is considerably larger, and the lattice and dispersion functions are considerably reduced. In all cases higher intensities and beam densities are expected.

Another feature is the different behavior of the transition energy and the chromaticity between the case of protons and that of the heavier ions. Whereas for heavier ions the chromaticity is about unit and the beam energy is well below the transition energy, for the case of protons the chromaticity is negative and small, and the transition energy $\gamma_{T}$ is less than unit. This could be an interesting beam-dynamics feature for some beam applications.

The CRFQ ion storage ring can be used for molecular and atomic physics and other lowenergy applications. The stored beam can be made to collide with another countermoving ion beam of the same or different species, with an electron beam, or with a photon beam (X-ray and ultraviolet radiation). Cooling techniques can also be applied, like electron and/or laser cooling. For this purpose the ring can be made of a racetrack shape. In this case, of course there is no external bending field in the straight sections. This device is ideal [7] for the demonstration of "Crystalline Beams". Moreover, in colliding beam mode, two intersecting storage rings, as shown in Figure 11, can be used to learn about nuclear fusion between protons and ions of boron [9].

Like in the previous example, one needs again to learn how to input the RF power into the device. Moreover, to reach the high intensity the device is capable of storing one needs to develop a method of multi-turn injection.

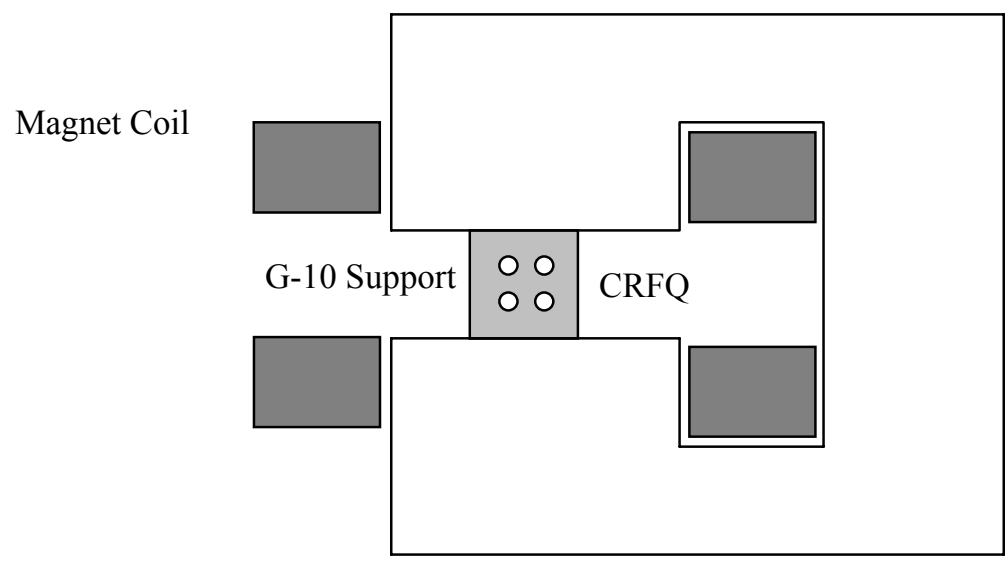

Figure 10. An Ion CRFQ Storage Ring within a Dipole Magnet 


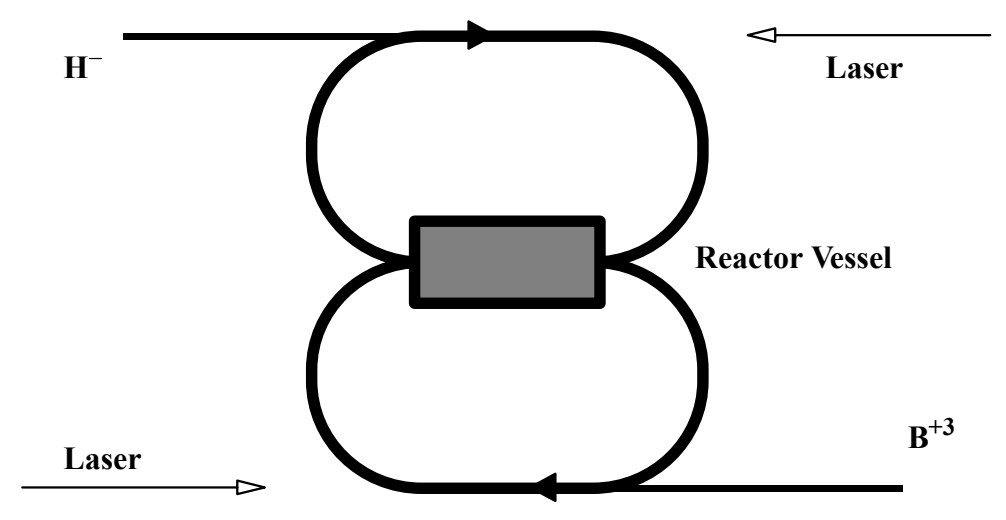

Figure 11. Colliding CRFQ Storage Rings [9]

\section{A FAA Application}

This is a CRFQ storage ring for the storage of $1.75-\mathrm{MeV}$ protons at an intensity of $10-$ $100 \mathrm{~mA}$. The main parameters are reported in Table 3 . The primary application of the storage ring is the production of intense and collimated $\gamma$-rays at about the same proton energy. The radiation is used for screening of objects and the examination of the contained, for instance at airports. For this purpose, a carbon foil with a thickness $\tau=1 \mu \mathrm{g} / \mathrm{cm}^{2}$ is inserted along the path of the beam. The following reaction occurs

$$
\mathrm{p}+{ }^{13} \mathrm{C} \rightarrow{ }^{14} \mathrm{~N}+\gamma
$$

The cross-section of the event $\sigma=1$ barn. With a similar reaction, and the same carbon target material, it is also possible to produce a beam of neutrons essentially thermal. Neutrons can also be used for medical and other industrial applications. In this case the proton energy is to be adjusted to $3.24 \mathrm{MeV}$. The case of neutron production is also shown in Table 3. It is found that it is necessary to add an external field to confine the beam within the coil aperture, and a lower excitation RF frequency.

Considering the nature of the application, we have intentionally taken a small and compact ring with a major radius $\mathrm{R}=75 \mathrm{~cm}$. The minor radius, $\mathrm{b}_{1}=1 \mathrm{~cm}$, is sufficiently large to accommodate the beam with a radius of the cross-section of at most $\mathrm{a}=2 \mathrm{~mm}$. At the same time, the beam angular divergence is $2.9 \mathrm{mrad}$ at $1.75 \mathrm{MeV}$, and $2.5 \mathrm{mrad}$ at $3.24 \mathrm{MeV}$. At $1.75 \mathrm{MeV}$ the bending field required for the exact cancellation of the curvature is $2.55 \mathrm{kG}$; at $3.24 \mathrm{MeV}$ it is $3.47 \mathrm{kG}$.

Since, as we shall see below, acceleration is required and the coils have thus convolution, we have adopted an integer value for the number of periods per turn $n_{s}=15$. For a given beam energy we could then determine the RF frequency that ranges between 58 and 79 $\mathrm{MHz}$. The required RF peak voltage correspondingly is 12 and $22 \mathrm{kVolt}$. 
In the following we shall concentrate to the case of $\gamma$-production $(1.75 \mathrm{MeV})$. The rate of production is given by

$\mathrm{dn}_{\gamma} / \mathrm{dt} \quad=\quad \tau \mathrm{I}_{\mathrm{p}} \sigma / \mathrm{e} \mathrm{m} \mathrm{m}_{\mathrm{p}} \mathrm{A}$

with $\mathrm{e}=1.6 \times 10^{-19} \mathrm{C}$ the electron charge, $\mathrm{m}_{\mathrm{p}}=1.67 \times 10^{-24} \mathrm{~g}$ the proton mass, and $\mathrm{A}=13$ the Carbon mass number. With $\tau=1 \mu \mathrm{g} / \mathrm{cm}^{2}$ and an average proton circulating intensity $\mathrm{I}=34 \mathrm{~mA}$, the production rate is $0.98 \times 10^{10} \mathrm{\gamma} / \mathrm{s}$. This corresponds to an instantaneous radiation level of 0.26 Curie. Every time a proton impinges on the target and $\mathrm{a} \gamma$ is emitted, it is of course lost. The corresponding beam loss rate is $\mathrm{dI}_{\mathrm{p}} / \mathrm{dt}=-1.6$ $\mathrm{nA} / \mathrm{s}$. Assuming a one-second long pulse, the beam intensity drop is of only $1.6 \mathrm{nA}$, and there is thus no need to refill continuously the proton beam.

Table 3. Parameters of the CRFQ for the FAA Application

\begin{tabular}{|l|r|r|}
\hline & $\gamma$-radiation & $\mathrm{n}$ - radiation \\
\hline \hline Kinetic Energy, $\mathrm{MeV}$ & 1.7476 & 3.2357 \\
\hline$\beta$ & 0.0609 & 0.0828 \\
$\gamma$ & 1.0019 & 1.0034 \\
\hline Momentum, $\mathrm{p}, \mathrm{MeV} / \mathrm{c}$ & 57.29 & 77.99 \\
\hline Mag. Rigidity, $\mathrm{kG}-\mathrm{m}$ & 1.911 & 2.601 \\
\hline Excitation $\mathrm{RF}, \mathrm{MHz}$ & 58.162 & 79.047 \\
\hline Wavelength, $\lambda, \mathrm{cm}$ & 515.4 & 379.3 \\
\hline Focus. Period, $\mathrm{L}, \mathrm{cm}$ & 31.42 & 31.42 \\
\hline Internal Radius, $\mathrm{b}_{1}, \mathrm{~mm}$ & 10 & 10 \\
\hline RF Voltage, $\mathrm{V}_{\mathrm{o}}, \mathrm{kV}$ & 12.05 & 22.29 \\
\hline Phase Adv. $/$ Period & $90^{\circ}$ & $90^{\circ}$ \\
\hline$\beta_{\mathrm{L}}-$ max, $\mathrm{cm}$ & 59.00 & 59.00 \\
\hline Major Radius, $\mathrm{R}, \mathrm{cm}$ & 75 & 75 \\
\hline Max. Dispersion, $\mathrm{cm}$ & 26.32 & 26.32 \\
\hline No. of Periods, $\mathrm{n}_{\mathrm{s}}$ & 15 & 15 \\
\hline Betatron Tune, $\nu_{\mathrm{s}}$ & 3.75 & 3.75 \\
\hline Transition Energy, $\gamma_{\mathrm{T}}$ & 1.838 & 1.838 \\
\hline Chromaticity & 0.9924 & 0.9859 \\
\hline Norm. Emitt., $\pi \mathrm{mm} \mathrm{mrad}$ & 0.30 & 0.30 \\
\hline Actual Emitt., $\pi \mathrm{mm} \mathrm{mrad}$ & 4.91 & 3.61 \\
\hline Beam Radius, $\mathrm{a}, \mathrm{mm}$ & 1.70 & 1.46 \\
\hline No. Protons, $\mathrm{N}, \mathrm{x} 10^{10}$ & 5.45 & 7.40 \\
\hline Revol. Frequency, MHz & 3.877 & 5.270 \\
\hline Beam Pulse Length, $\mu \mathrm{s}$ & 0.193 & 0.142 \\
\hline Beam Current, $\mathrm{I}_{\mathrm{p}}, \mathrm{mA}$ & 33.8 & 62.4 \\
\hline
\end{tabular}

At the moment of production, the $\gamma$-ray spot size and divergence are equal to those of the incident proton beam, that is, $1.7 \mathrm{~mm}$ and $2.9 \mathrm{mrad}$. If the object to be irradiated and 
screened is located at a distance of $1.5 \mathrm{~m}$, the $\gamma$-ray spot size on it has a diameter of $1 \mathrm{~cm}$. Thus, the $\gamma$-ray is well collimated and intense for the proposed FAA application. Protons that do not produce $\gamma$ 's, continuously traversing the target turn-after-turn, undergo to multiple Coulomb scattering with the atoms of Carbon in the foil. This causes an increase of the beam divergence, and thus of the beam emittance, a systematic energy loss, and an increase of the energy spread. The photo-production is a resonance reaction with a very narrow peak. The width of the resonance is about $150 \mathrm{eV}$; therefore, the beam energy spread is to be about $0.01 \%$ or less at all time if all the protons are to be involved in the $\gamma$-production. The systematic energy loss is about $150 \mathrm{eV}$ per target crossing. The beam would lose all its energy in only ten thousand turns. Moreover, it takes only few revolutions for the beam to exit the resonance condition. There is, thus, need to compensate instantaneously the energy loss. This is done with RF acceleration that just preserves the beam energy to the reference value.

To provide acceleration the coil are to be convoluted internally. Moreover, the number of period $n_{s}$ per turn is to be exactly an integer. According to Eq. (78), the energy gain per turn is

$\Delta \mathrm{E}=\pi \mathrm{n}_{\mathrm{s}} \mathrm{eV}_{\mathrm{o}} \xi \sin \phi_{\mathrm{s}}$

$\xi=\left(b_{2}-b_{1}\right) / b_{1}$ defines the ratio of the convolution internal diameters. Clearly a small $\mathrm{RF}$ phase angle is required. We take $\phi_{\mathrm{s}}=0.15^{\circ}$. For $\Delta \mathrm{E}=150 \mathrm{eV} / \mathrm{turn}$, a relatively small convolution is required, $\mathrm{b}_{2}-\mathrm{b}_{1}=1 \mathrm{~mm}$.

But energy straggling is a problem. Every time the beam traverses the carbon target there is an rms energy spread increase of $270 \mathrm{eV}$ that of course adds only quadratically turnafter-turn, but it is still too large compared to the resonance width of about $150 \mathrm{eV}$. At the same time, also the emittance increase due to multiple scattering is a concern. The estimate is an increase of the rms emittance of $0.005 \mathrm{~mm}-\mathrm{mrad}$ per turn. The beam emittance would thus double in value after about ten thousand revolutions ( $\sim 3 \mathrm{~ms})$.

The device has a layout similar to the one show in Figure 8, with the exception that the linear RFQ accelerates to $1.75 \mathrm{MeV}$ (or $3.24 \mathrm{MeV}$ ) and that, together to the matching linear section, operates at the same frequency adopted for the CRFQ. Provided that the ion source has the intensity capability, only a short single-turn beam pulse is injected per cycle. The duty cycle can be reasonably low so that water-cooling and radiation shielding can be eliminated or, at least, minimized. The recommended mode of operation is to pulse the device at a reasonable low repetition rate, with a pulse period short enough to minimize the effects on the beam quality deterioration. A reasonable and feasible choice is a repetition rate of 60 pulses per second, each pulse having a period of few milliseconds. Even a low duty cycle of only one percent still gives a sufficient amount of $\gamma$-radiation: an average $3 \mathrm{mCi}$. 


\section{A Medical Application}

This is a compact accelerator for the acceleration of protons from 20 to $250 \mathrm{MeV}$. The required repetition rate is 60 pulses per second and the beam intensity is $10^{9}$ protons per pulse. There is an external bending field that is also ramped with the beam energy during acceleration. The four coils are convoluted to create an accelerating longitudinal field. The main parameters at injection and extraction energy are given in Table 4. The radius of the accelerator $\mathrm{R}=250 \mathrm{~cm}$. The RFQ parameter $\mathrm{B}$ is constant during the acceleration cycle and adjusted for a phase advance per period of $90^{\circ}$. There are exactly $n_{s}=13$ focusing periods. The magnet that creates the external bending field, to cancel the local curvature, covers the entire circumference of the CRFQ accelerator. The magnetic field ranges between $2.60 \mathrm{kG}$ at injection and $9.73 \mathrm{kG}$ at extraction. Considering the high repetition rate, a sinusoidal excitation of the guiding field is assumed. The intensity of $10^{9}$ protons per pulse is modest and well below the space-charge limit. It is to be observed that the lattice and dispersion functions are constant during the acceleration cycle, but that the chromaticity varies. The beam energy during the entire acceleration cycle is below the transition energy.

Table 4. A CRFQ Accelerator for Medical Application

\begin{tabular}{|l|r|r|}
\hline & Injection & Extraction \\
\hline \hline Kinetic Energy, MeV & 20 & 250 \\
\hline$\beta$ & 0.2032 & 0.6136 \\
\hline$\gamma$ & 1.02132 & 1.2665 \\
\hline Momentum, MeV/c & 194.8 & 729.1 \\
\hline Magnetic Rigidity, $\mathrm{kG}-\mathrm{m}$ & 6.496 & 24.321 \\
\hline Bending Field, $\mathrm{kG}$ & 2.60 & 9.73 \\
\hline RF Excitation, $\mathrm{MHz}$ & 50.43 & 152.24 \\
\hline Wavelength, $\lambda, \mathrm{cm}$ & 594.5 & 196.9 \\
\hline Period Length, $\mathrm{L}, \mathrm{cm}$ & 120.83 & 120.83 \\
\hline Inner Coil Radius, $\mathrm{b}_{1}, \mathrm{~cm}$ & 1.0 & 1.0 \\
\hline Outer Coil Radius, $\mathrm{b}_{2}, \mathrm{~cm}$ & 1.2 & 1.2 \\
\hline RF Peak Voltage, $\mathrm{kV}$ & 11.54 & 130.41 \\
\hline$\beta-$ max, cm & 226.92 & 226.92 \\
\hline Dispersion $-\mathrm{max}, \mathrm{cm}$ & 116.8 & 116.8 \\
\hline No. of Periods, $\mathrm{n}_{\mathrm{s}}$ & 13 & 13 \\
\hline Betatron Tune, $v_{\mathrm{s}}$ & 3.25 & 3.25 \\
\hline Transition Energy, $\gamma_{\mathrm{T}}$ & 1.593 & 1.593 \\
\hline Chromaticity & 0.9152 & 0.2273 \\
\hline Norm. Emitt., $\pi \mathrm{mm}-\mathrm{mrad}$ & 0.30 & 0.30 \\
\hline Act. Emitt., $\pi \mathrm{mm}-\mathrm{mrad}$ & 1.445 & 0.386 \\
\hline Beam Radius, a, mm & 1.811 & 0.936 \\
\hline Rev. Frequency, MHz & 3.879 & 11.711 \\
\hline Tot. No. of Protons, $\mathrm{x} 10^{9}$ & 1 & 1 \\
\hline Ave. Beam Current, $\mathrm{mA}$ & 0.62 & 1.87 \\
\hline
\end{tabular}


In order to provide for acceleration several conditions are to be satisfied. First of all, the period length, $L=\beta \lambda$, has to be kept constant and integer. As the beam is accelerated its energy and velocity vary. Consequently, the RF wavelength has also to vary as $\lambda=\mathrm{L} / \beta$. The RF frequency will vary accordingly as $\mathrm{c} / \lambda$. In the example of the medical accelerator described in Table 4 , the ratio of changes of $\beta, \lambda$ and RF frequency is about three.

During the acceleration cycle, the RFQ parameter B, Eq. (18), is also kept constant to preserve the same lattice functions and phase advance per period. Consequently, the peak $\mathrm{RF}$ voltage varies with the beam energy as follows $(\mathrm{A}=\mathrm{Q}=1)$

$\mathrm{eV}_{\mathrm{o}}=\mathrm{mc}^{2} \mathrm{~b}_{1}^{2} \mathrm{~B} \gamma \beta^{2} / 2(1-\xi) \mathrm{L}^{2}$

with $\xi=\left(b_{2}-b_{1}\right) / b_{1}=0.2$. As shown in Table 4 , the range of variation of the peak voltage $\mathrm{V}_{\mathrm{o}}$ during the acceleration cycle is between $11.54 \mathrm{kV}$ at injection and $130.41 \mathrm{kV}$ at extraction. At the same time, the energy gain per turn, as function of the beam energy during the acceleration cycle, is given by

$\Delta \mathrm{E}=\mathrm{mc}^{2} \mathrm{~b}_{1}^{2} \mathrm{~B} \mathrm{n}_{\mathrm{s}}{ }^{3} \xi\left(\sin \phi_{\mathrm{s}}\right)\left(\beta^{2} \gamma\right) / 8 \pi \mathrm{R}^{2}(1-\xi)$

The external bending field, $B_{\text {ext }}$ is adjusted to cancel exactly the local curvature. Moreover, it follows its own variation with time that, as we have said, we take to be sinusoidal. Denoting with $\mathrm{T}$ the cycle period

$\mathrm{B}_{\mathrm{ext}}=\mathrm{B}_{1}-\mathrm{B}_{2} \cos (2 \pi \mathrm{t} / \mathrm{T})$

The beam momentum $\mathrm{p}$ has to follow the variation of the bending field according to

$\mathrm{pc} \quad=\quad 1.0 \times 10^{-7} \mathrm{c} \mathrm{R} \mathrm{B}_{\mathrm{ext}}$

with $\mathrm{pc}=\beta \gamma \mathrm{mc}^{2}$ in $\mathrm{MeV}, \mathrm{R}$ in meter, the speed of light $\mathrm{c}$ in $\mathrm{m} / \mathrm{s}$, and $\mathrm{B}_{\text {ext }}$ in $\mathrm{kGauss}$. Also the energy gain per turn has to follow the variation of the bending field according to

$\Delta \mathrm{E}=2.0 \times 10^{-4} \pi \mathrm{R}^{2}\left(\mathrm{~dB}_{\mathrm{ext}} / \mathrm{dt}\right)$

with $\Delta \mathrm{E}$ in $\mathrm{keV} /$ turn, $\mathrm{R}$ in meter, and $\mathrm{dB}_{\text {ext }} / \mathrm{dt}$ in $\mathrm{kGauss} / \mathrm{s}$. Finally, combining Eqs. (96, 98 and 99) yields the following relation between the RF phase angle $\phi_{\mathrm{s}}$ and the guiding field $\mathrm{B}_{\text {ext }}$, that is to be satisfied during the acceleration cycle,

$\sin \phi_{\mathrm{s}}=\quad 16 \pi^{2} \mathrm{R}^{3}(1-\xi)\left(\mathrm{dB}_{\mathrm{ext}} / \mathrm{dt}\right) / \mathrm{B} \mathrm{B}_{\mathrm{ext}} \mathrm{c} \beta \xi \mathrm{b}_{1}{ }^{2} \mathrm{n}_{\mathrm{s}}{ }^{3}$

Knowing the RF phase angle $\phi_{\mathrm{s}}$ and amplitude $\mathrm{V}_{\mathrm{o}}$ it is possible then to estimate the area and the height of the RF buckets and the longitudinal oscillation frequency in the usual way. The RF peak voltage $V_{o}$ and $\sin \phi_{s}$ for our example of medical accelerator are displayed in Figure 12 and 13 respectively. 


\section{The CRFQ Accelerator}

It is possible to operate the CRFQ also at large beam energies when $\beta \sim 1$. Also in this case there are considerable advantages over the conventional magnetic rings but also some peculiar behavior of the lattice appears. To illustrate the possible application of the CRFQ as a high-energy accelerator we shall consider two cases. One is a proton accelerator of $30 \mathrm{GeV}$ that assumes a $1.5 \mathrm{GeV}$ injector, for instance, a Super-Conducting Linac. We shall require a beam intensity of $10^{12}$ protons per pulse at the repetition rate of one beam pulse every few seconds. As a practical example, we assume a linear ramp of the external bending field over a period of 1 second. This accelerator can be used, for instance, as the source for Proton Radiography [10]. The second example is an electron accelerator of $800 \mathrm{MeV}$ with injection at $50 \mathrm{MeV}$. Also here the intensity is $10^{12}$ electrons per pulse; but the repetition rate can be higher, for instance 15 pulses per second. For this purpose, the ramp of the bending field is taken to be sinusoidal. The main parameters for the two accelerators are given in Table 5 .

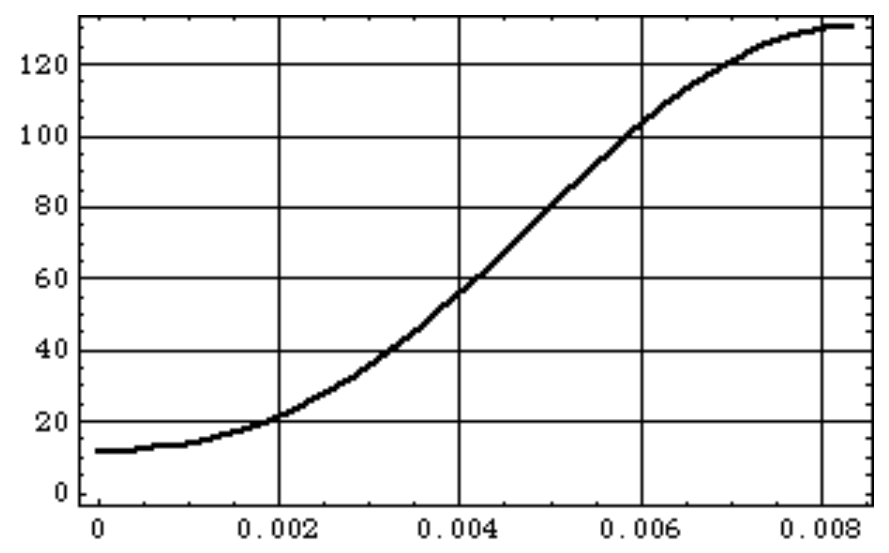

Figure 12. The RF Voltage $\mathrm{V}_{\mathrm{o}}(\mathrm{kV} /$ turn) vs. time (s) during the acceleration cycle

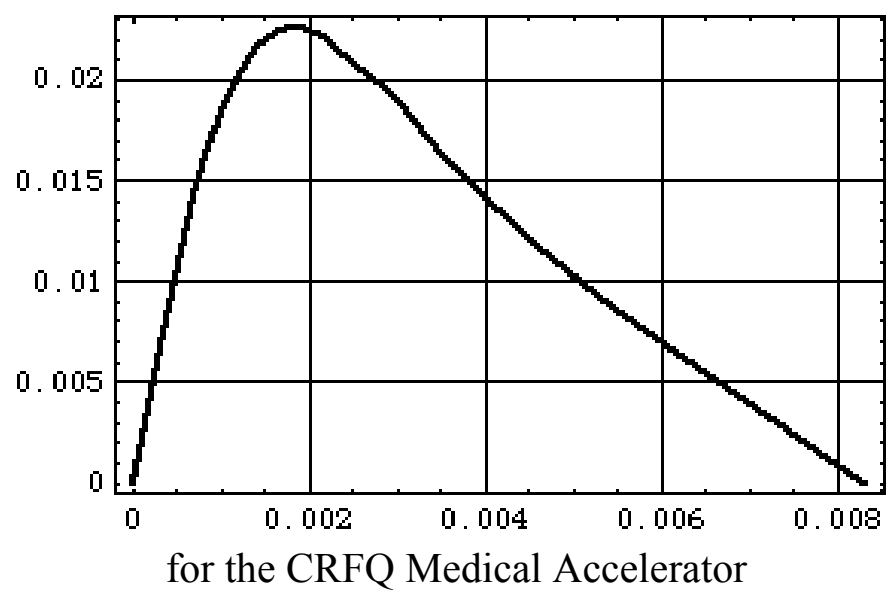

Figure 13. $\sin \phi_{\mathrm{s}}$ vs. time (s) during the acceleration cycle for the CRFQ Medical Accelerator 
The design considerations for the proton accelerator are similar to those we have described for our medical accelerator model. The RF voltage $\mathrm{V}_{\mathrm{o}}$ varies about linearly with time during the acceleration cycle. The RF phase program is shown in Figure 14. If indeed the CRFQ has a higher space-charge limit, it would be the major advantage for the high-energy application. The intensity of $10^{12}$ protons is still well below the limit given by Eqs. (56 and 57) also with a small beam emittance; we have thus adopted a phase advance per period of $60^{\circ}$ that requires $B=4.6023$. The beam radius is $1.75 \mathrm{~mm}$ at injection and $0.47 \mathrm{~mm}$ at extraction. In our example, to avoid an exceedingly large RF voltage, we have adopted a small number of long periods, giving lattice parameters similar to those of conventional magnetic accelerators. Nevertheless, the transition energy is located somewhere in between injection and extraction. Crossing the transition energy in the CRFQ accelerator may present interesting beam performance issues to be explored. At the same time, the chromaticity is very large and negative with unpredictable consequences that need to be investigated in more details.

Table 5. CRFQ High-Energy Accelerators

\begin{tabular}{|l|r|r|r|r|}
\hline & \multicolumn{2}{|c|}{ Protons } & \multicolumn{2}{c|}{ Electrons } \\
\hline & Injection & Extraction & Injection & Extraction \\
\hline \hline Kinetic Energy, GeV & 1.5 & 30 & 0.050 & 0.300 \\
\hline$\beta$ & 0.9230 & 0.9995 & 0.99995 & 1.00000 \\
\hline$\gamma$ & 2.599 & 32.974 & 98.847 & 1566.56 \\
\hline Momentum, GeV/c & 2.25 & 30.92 & 0.05051 & 0.80051 \\
\hline Magnetic Rigidity, T-m & 7.507 & 103.15 & 0.1685 & 2.670 \\
\hline Bending Field, Tesla & 0.100 & 1.375 & 0.0674 & 1.068 \\
\hline RF Excitation, MHz & 27.60 & 29.89 & 209.93 & 209.94 \\
\hline Wavelength, $\lambda, \mathrm{m}$ & 10.86 & 10.03 & 1.428 & 1.428 \\
\hline Period Length, $\mathrm{L}, \mathrm{m}$ & 10.026 & 10.026 & 1.428 & 1.428 \\
\hline Inner Coil Radius, $\mathrm{b}_{1}, \mathrm{~cm}$ & 1.0 & 1.0 & 1.0 & 1.0 \\
\hline Outer Coil Radius, $\mathrm{b}_{2}, \mathrm{~cm}$ & 1.2 & 1.2 & 1.2 & 1.2 \\
\hline Major Radius, $\mathrm{R}, \mathrm{m}$ & 75 & 75 & 2.50 & 2.50 \\
\hline RF Peak Voltage, $\mathrm{kV}$ & 5.94 & 88.4 & 7.15 & 112.9 \\
\hline$\beta-$ max, $\mathrm{m}$ & 25.57 & 25.57 & 3.50 & 3.50 \\
\hline Dispersion $-\mathrm{max}, \mathrm{m}$ & 5.58 & 5.58 & 3.40 & 3.40 \\
\hline No. of Periods, $\mathrm{n}_{\mathrm{s}}$ & 47 & 47 & 11 & 11 \\
\hline Betatron Tune, $\mathrm{v}_{\mathrm{s}}$ & 7.833 & 7.833 & 1.833 & 1.833 \\
\hline Transition Energy, $\gamma_{\mathrm{T}}$ & 3.965 & 3.965 & 0.928 & 0.928 \\
\hline Chromaticity & -0.7483 & -1.0503 & -1.0520 & -1.0522 \\
\hline Norm. Emitt., $\pi \mathrm{mm-mrad}$ & 0.30 & 0.30 & 3.0 & 3.0 \\
\hline Act. Emitt., $\pi$ mm-mrad & 0.125 & 0.0091 & 0.030 & 0.0019 \\
\hline Beam Radius, $\mathrm{a}, \mathrm{mm}$ & 1.75 & 0.473 & 0.326 & 0.082 \\
\hline Rev. Frequency, MHz & 0.587 & 0.636 & 19.08 & 19.08 \\
\hline Tot. No. of Particles, $\mathrm{x} 10{ }^{12}$ & 1 & 1 & & 1 \\
\hline Ave. Beam Current, Amp & 0.094 & 0.102 & 3.05 & 3.05 \\
\hline Space-Charge Parameter $\Delta$ & 0.0130 & 0.0120 & 0.0051 & 0.0051 \\
\hline
\end{tabular}


In the case of the electron accelerator the excitation RF frequency is constant during the acceleration cycle and equal to about 209.9 MHz. Also in this case it is expected that the beam intensity is considerably below the CRFQ space-charge limit and, as a consequence we have adopted again a phase advance per period of $60^{\circ}$. In contrast, the behavior of the chromaticity is not problematic. At all times the beam energy is well above the transition energy. The major difference is that electrons lose energy to synchrotron radiation. In the case under consideration an electron loses at most $14.5 \mathrm{keV}$ per turn at top energy. At that time the power lost is about $60 \mathrm{~kW}$ that is given back by the same accelerating RF system.

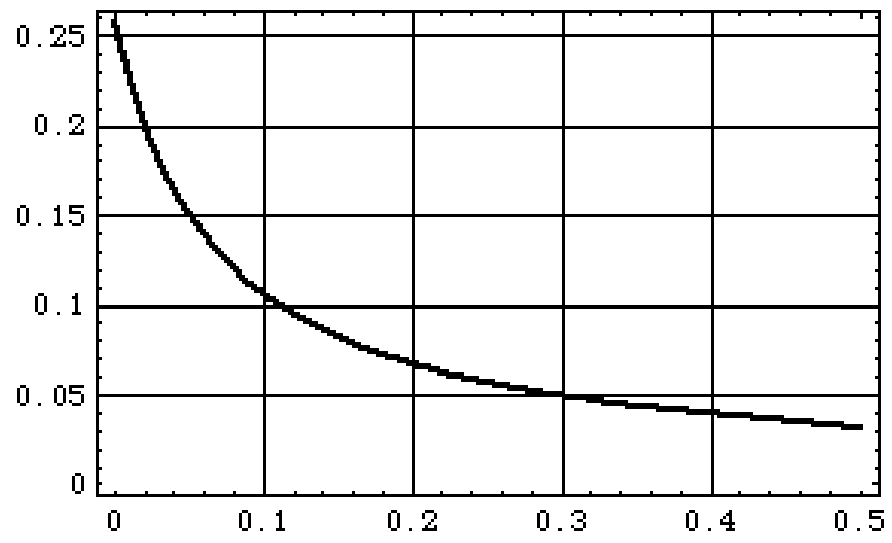

Figure 14. $\sin \phi_{\mathrm{s}}$ vs. time (s) during the acceleration cycle for the CRFQ Proton Accelerator

To estimate the RF acceleration cycle, Eq. (99), that gives the energy gain per turn, is also modified to include a term for the energy loss

$\Delta \mathrm{E}=2.0 \times 10^{-4} \pi \mathrm{R}^{2}\left(\mathrm{~dB}_{\mathrm{ext}} / \mathrm{dt}\right)+\mathrm{U}_{\mathrm{rad}}$

with

$\mathrm{U}_{\mathrm{rad}}=(88.5 \mathrm{keV} / \mathrm{turn}) \mathrm{E}_{\mathrm{GeV}}{ }^{4} / \mathrm{R}_{\text {meter }}$

The RF Voltage $V_{o}$ and $\sin \phi_{\mathrm{s}}$ for the electron accelerator are shown in Figures 15 and 16. 


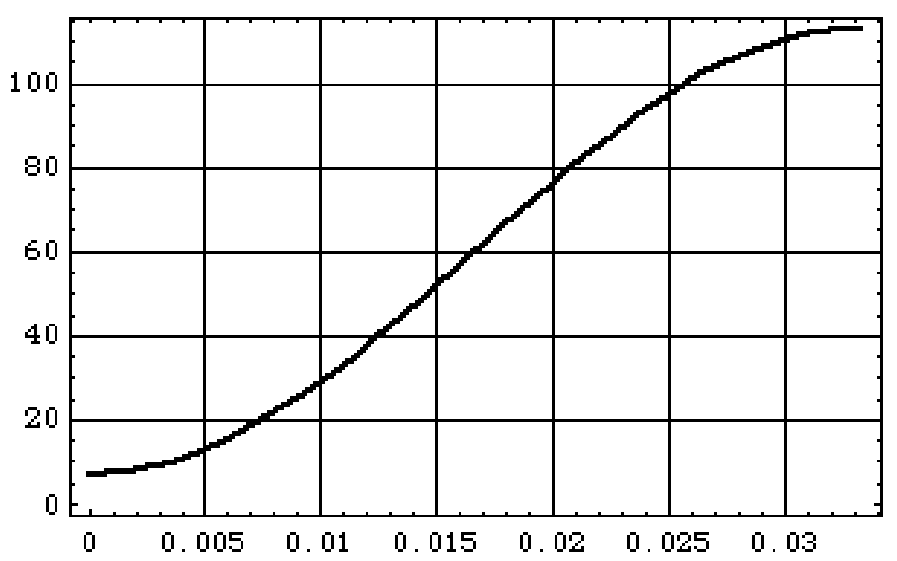

Figure 15. The RF Voltage $\mathrm{V}_{\mathrm{o}}(\mathrm{kV} /$ turn) vs. time (s) during the acceleration cycle for the CRFQ Electron Accelerator

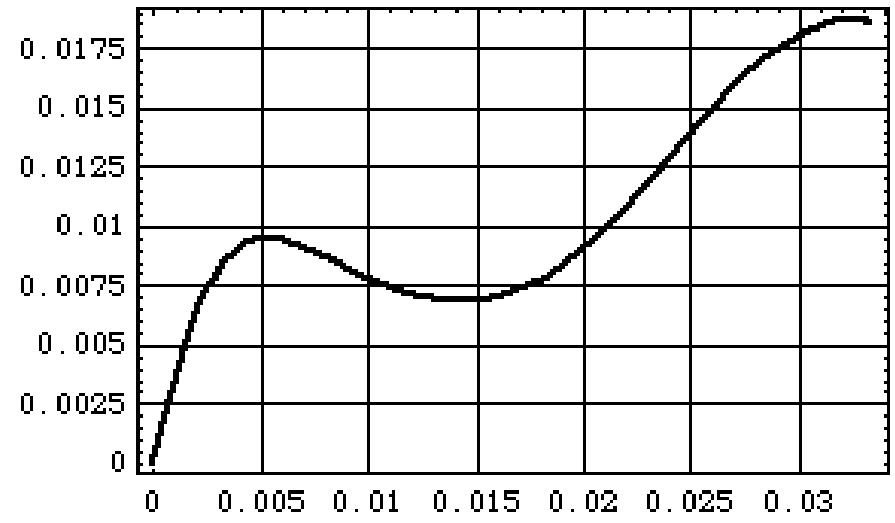

Figure 16. $\sin \phi_{\mathrm{s}}$ vs. time (s) during the acceleration cycle for the CRFQ Electron Accelerator

If the CRFQ has the racetrack shape of Figure 6, it is sufficient to provide acceleration to shape the rods with convolution only in the straight sections and leave the rods smooth in the arcs. With the proper length of the straight accelerating sections is then possible to adjust the convolution depth $\xi$ to a technically more feasible value. Of course, in this case one should insure rational relation between period length $\mathrm{L}$, straight section length $\mathrm{H}$, and circumference $2 \pi R$ so that particles enter the accelerating straights always in phase with the RF.

\section{Compact Synchrotron Radiation Facility}

The CRFQ can be used as a compact storage ring for synchrotron radiation. There are some special features that make the application peculiar and interesting. First of all, the arrangement of the four rods (shown in Figures 1 and 2) rotates the transverse modes of oscillation to $45^{\circ}$ with the normal axis $\mathrm{x}$ and $\mathrm{z}$, as shown in Figure 3. Aside from a longitudinal shift of $180^{\circ}$, the amplitude lattice function $\beta_{\mathrm{L}}$ and dispersion $\eta$ are exactly 
equal for the two modes of oscillation. The excitation of quantum fluctuation is also the same for the two modes and, as a result, the beam shape is everywhere about round with the same cross-section radius along the horizontal and vertical direction. We shall first consider the case that an external bending field is added to cancel exactly the local curvature.

We remind that the energy loss per electron and per turn is

$$
\mathrm{U}=(88.5 \mathrm{keV} / \text { turn }) \mathrm{E}_{\mathrm{GeV}}{ }^{4} / \mathrm{R}_{\text {meter }}
$$

The critical radiation wavelength

$$
\lambda_{\mathrm{c}}=\left(18.6 \mathrm{~A}^{\circ}\right) / \mathrm{B}_{\mathrm{ext}}(\text { Tesla }) \mathrm{E}_{\mathrm{GeV}}^{2}
$$

The repartition factors

$$
\begin{array}{lll}
\mathrm{J}_{0} & =16.776(\mathrm{~L} / \pi \mathrm{R})^{2} \\
\mathrm{~J}_{\mathrm{E}} & =2+\mathrm{J}_{0} \\
\mathrm{~J}_{\mathrm{uv}} & =1-\mathrm{J}_{0}
\end{array}
$$

The damping times, along the $(\mathrm{u}, \mathrm{v})$ normal modes of oscillation,

$$
\tau_{\text {Euv }}=(0.476 \mathrm{~ms}) \mathrm{R}_{\mathrm{m}}^{2} / \mathrm{J}_{\mathrm{Euv}} \mathrm{E}_{\mathrm{GeV}}{ }^{3}
$$

The equilibrium rms energy spread

$\sigma_{\mathrm{E}} / \mathrm{E}=0.62 \times 10^{-6} \gamma /\left(\mathrm{J}_{\mathrm{E}} \mathrm{R}_{\mathrm{m}}\right)^{1 / 2}$

The equilibrium rms emittance $\left(\sigma^{2} / \beta_{\mathrm{L}}\right.$, along the $(\mathrm{u}, \mathrm{v})$ normal modes of oscillation, in $\mathrm{mm}-\mathrm{mrad} \equiv \mu \mathrm{m})$

$$
\varepsilon_{\mathrm{uv}}=(0.374 \mu \mathrm{m}) \gamma^{2} \mathrm{~J}_{0}^{3 / 2} /\left(1-\mathrm{J}_{0}\right)
$$

Thus the equilibrium emittance is proportional to $(\mathrm{L} / \mathrm{R})^{3}$. It is possible to obtain a very brilliant radiation source with a large size ring having a small period. An example is given in the following Table 6 . The phase advance per period has been set to $90^{\circ}$.

The beam dimensions and intensity shown in Table 6 correspond to the case when the effects of the synchrotron radiation are neglected. That is, the beam dimension and intensity depend only on the injector performance. The beam values in equilibrium with synchrotron radiation are summarized in Table 7 . The case in question has the same beam size with or without the effect of the synchrotron radiation. As consequence, the beam intensity is at the space charge limit in both cases. A large RF frequency has been adopted, with a significant large RF voltage. It would be desirable to reduce the internal dimensions of the CRFQ to the point that tolerances become very tight. Moreover, the RF phase is small because the actual longitudinal field is very large compared to the amount 
of energy lost per turn. The control of the RF phase is also a point of concern. The case we have illustrated is just an example not necessarily optimized. Other cases may be possible.

It is seen that in the case $\mathrm{J}_{0}<<1$, equivalent to $\mathrm{L} / \mathrm{R}<<0.77$, the normalized equilibrium emittance is given by

$\gamma \varepsilon_{\mathrm{uv}}=(168 \mu \mathrm{m}) \mathrm{B}_{\mathrm{ext}}^{3} \lambda^{3}$

with $B_{\text {ext }}$ in Tesla and the RF wavelength $\lambda$ in meter. For instance, by letting $B_{\text {ext }}=1$ Tesla and $\lambda=20 \mathrm{~cm}$, corresponding to a RF frequency of $1.5 \mathrm{GHz}$, the normalized emittance $\gamma \varepsilon_{\mathrm{uv}} \sim 1 \mu \mathrm{m}$. This result is independent of beam energy.

Table 6. CRFQ Synchrotron Radiation Facility

\begin{tabular}{|l|r|}
\hline Kinetic Energy, MeV & 800 \\
\hline \hline$\beta$ & 1.00000 \\
\hline$\gamma$ & 1566.56 \\
\hline Momentum, MeV/c & 800.51 \\
\hline Magnetic Rigidity, kG-m & 26.702 \\
\hline Bending Field, kGauss & 10.68 \\
\hline RF Excitation, MHz & 1202.4 \\
\hline Wavelength, $\lambda, \mathrm{cm}$ & 24.933 \\
\hline Period Length, $\mathrm{L}, \mathrm{m}$ & 24.933 \\
\hline Inner Coil Radius, $\mathrm{b}_{1}, \mathrm{~cm}$ & 0.5 \\
\hline Outer Coil Radius, $\mathrm{b}_{2}, \mathrm{~cm}$ & 0.6 \\
\hline Major Radius, $\mathrm{R}, \mathrm{m}$ & 2.50 \\
\hline RF Peak Voltage, $\mathrm{MV}$ & 1.370 \\
\hline$\beta-$ max, cm & 46.8 \\
\hline Dispersion - max, cm & 4.973 \\
\hline No. of Periods, $\mathrm{n}_{\mathrm{s}}$ & 63 \\
\hline Betatron Tune, $v_{\mathrm{s}}$ & 15.75 \\
\hline Transition Energy, $\gamma_{\mathrm{T}}$ & 7.72 \\
\hline Chromaticity & -1.0522 \\
\hline Norm. Emitt., $\pi \mathrm{mm}-\mathrm{mrad}$ & 3.0 \\
\hline Act. Emitt., $\pi \mathrm{mm}-\mathrm{mrad}$ & 0.0019 \\
\hline Beam Radius, $\mathrm{a}, \mu \mathrm{m}$ & 29.9 \\
\hline Rev. Frequency, MHz & 19.085 \\
\hline Tot. No. of Particles, $\mathrm{x} 10^{10}$ & 2 \\
\hline Ave. Beam Current, Amp & 0.0611 \\
\hline Space-Charge Parameter $\Delta$ & 0.0429 \\
\hline
\end{tabular}


Table 7. CRFQ Synchrotron Radiation Parameters

\begin{tabular}{|l|r|}
\hline Kinetic Energy, MeV & 800 \\
\hline \hline Energy Loss/Turn, keV & 14.5 \\
\hline Critical Wavelength $\lambda_{\mathrm{o}}, \mathrm{A}^{\mathrm{o}}$ & 27.21 \\
\hline Critical Energy, keV & 0.455 \\
\hline Flux, photons / s mrad 1\%BW & $8.0 \times 10$ \\
\hline $\mathrm{J}_{0}$ & 0.0169 \\
\hline $\mathrm{J}_{\mathrm{E}}$ & 2.0169 \\
\hline $\mathrm{J}_{\mathrm{XZ}}$ & 0.9831 \\
\hline Damping Time, $\tau_{\mathrm{E}}, \mathrm{ms}$ & 2.88 \\
\hline Damping Time, $\tau_{\mathrm{XZ}}, \mathrm{ms}$ & 5.91 \\
\hline Energy Spread, $\sigma_{\mathrm{E}} / \mathrm{E}, \%$ & 0.0432 \\
\hline rms Emittance, $\mathrm{mm}-\mathrm{mrad}$ & 0.00205 \\
\hline Equil. Beam Radius, a, $\mu \mathrm{m}$ & 31.00 \\
\hline rf Phase, $\phi_{\mathrm{s}}$, degrees & 0.0153 \\
\hline Tot. No. of Particles, $\mathrm{x} 10{ }^{10}$ & 2 \\
\hline Ave. Beam Current, Amp & 0.0611 \\
\hline Space-Charge Parameter $\Delta$ & 0.043 \\
\hline
\end{tabular}

\section{Engineering}

There are several engineering issues for the design of a CRFQ storage-accelerator ring that need to be investigated. Outstanding issues are listed below.

- Mechanical engineering and design of the ring

- How to input RF power in the device.

- Design of the RF Cavities.

- Injection of a short single-turn beam pulse.

- Multi-turn injection of a long beam pulse.

- Design of the Matching Section between Injector and the CRFQ.

- Beam diagnostic and instrumentation.

- Vacuum requirements.

- Power requirements and considerations.

- Design of the external Bending Magnet.

- Intra-beam scattering in the high intensity mode of a single beam.

- Beam-beam scattering of two colliding beams, and beam-beam interaction. 


\section{Error Analysis}

The error analysis is somewhat different from that of conventional, magnetic storage and accelerator rings. Some issues for further investigation are:

- Control of the level and quality of the external bending field.

- RF Excitation and distribution of the RF field around the ring.

- Coil alignment.

\section{Conclusions}

We have investigated the motion of particles in a Circular RFQ (CRFQ) storage and accelerator ring. We believe that the CRFQ ring described in this paper represents a new challenging product of the accelerator technology that can have several useful and interesting applications. The CRFQ ring has with no doubt several advantages over the conventional magnetic accelerators, in summary: compactness, high beam intensity, small beam dimension, and less susceptibility to errors.

\section{References}

[1] A. G. Ruggiero, "The Circular RFQ Storage Ring". Proceedings of the Workshop on Nonlinear and Collective Phenomena in Beam Physics. Arcidosso, Italy, sept. 1998. Editors S. Chattopadhyay, M. Cornacchia, C. Pellegrini. AIP Conf. Proc. 468, p. 244.

[2] A. G. Ruggiero, " The Circular RFQ Storage Ring” . Proceedings of 1999 Particle Accelerator Conference, Vol. 5, p. 3731. New York City, NY. March - April, 1999.

[3] Moon and Spencer, Field Theory Handbook, Springer-Verlag. Berlin 1961.

[4] J. W. Staples, "RFQ's - An Introduction". The Physics of Particle Accelerators. AIP Conference Proceedings 249 (1992). Vol. II, p. 1483.

[5] Several problems discussed in the report have been solved by programming with Mathematica from Wolfram Research.

[6] E.D. Courant and H.S. Snyder, Annals of Physics, Vol. 3, 48 (1958).

[7] A.G. Ruggiero, "Crystalline Beams". Proceedings of the $2^{\text {nd }}$ ICFA Workshop on the Physics of High Brightness Beams. Page 124. Nov. 9-12,1999. UCLA, Los Angeles, Calif. USA. World Scientific. Editors: J. Rosenzweig and L. Serafini.

[8] F. Sacherer, Proceedings of the 1971 PAC, Chicago, Illinois. Page 1105.

[9] A.G. Ruggiero, "Proton-Boron Colliding Beams for Nuclear Fusion". ICONE-8095. Proceedings of ICONE-8, April 2-6, 2000, Baltimore, MD USA.

[10] The Scrounge-atron, A Proton Radiography Demonstration Accelerator. UCRL-LR-134107. December 18, 1998. 


\section{Appendix A}

In the main toroidal coordinate system, the wave equation Eq. (1) is

$$
\begin{aligned}
& \partial(A(\eta, \theta) \partial \mathrm{V} / \partial \eta) / \partial \eta+\partial(A(\eta, \theta) \partial \mathrm{V} / \partial \theta) / \partial \theta+ \\
& +\mathrm{R}^{2} \mathrm{q}^{2} \beta(\eta, \theta) \mathrm{V} \quad=0
\end{aligned}
$$

where [3]

$A(\eta, \theta)=\sinh \eta /(\cosh \eta-\cos \theta)$,

$\beta(\eta, \theta)=\sinh \eta /(\cosh \eta-\cos \theta)^{3}$.

In the region $\rho<b$ between the rods, $\eta$ is very large, and in particular $\eta=\infty$ in proximity of the circumference with radius $\mathrm{R}$. Thus, in good approximation, $\sinh \eta \sim \cos \eta>>$ $\cos \theta$, and $A(\eta, \theta) \sim 1$.

In the cylindrical coordinate system, a toroidal surface is described by the equation

$r^{2}+z^{2}+R^{2}=2 R r \operatorname{coth} \eta$

or, in the reduced toroidal system,

$\operatorname{coth} \eta=1+\rho^{2} / 2 R(R+x)$

In the region $\rho<\mathrm{b}$ between the rods, $\mathrm{e}^{\eta} \sim 2 \mathrm{R} / \rho, \partial / \partial \eta \sim-\rho \partial / \partial \rho$, and $\partial^{2} / \partial \eta^{2} \sim \rho^{2} \partial^{2} / \partial \rho^{2}+\rho \partial / \partial \rho$.

At the same time $\beta \sim 4 \mathrm{e}^{-2 \eta} \sim \rho^{2} / \mathrm{R}^{2}$.

By transforming to the reduced toroidal coordinate system, and by retaining only the lowest order terms, we finally derive Eq. (2). 


\section{Appendix B}

The electric $\mathbf{E}$ and magnetic $\mathbf{B}$ field vector components can be expressed as usual in terms of scalar $\mathrm{V}$ and vector $\mathbf{A}$ potentials

$$
\begin{aligned}
& \mathbf{E}=-\operatorname{grad} \mathrm{V}-(1 / \mathrm{c}) \partial \mathbf{A} / \partial \mathrm{t} \\
& \mathbf{B}=\operatorname{rot} \mathbf{A}
\end{aligned}
$$

related to each other by the Lorentz condition

$\operatorname{div} \mathbf{A}+(1 / \mathrm{c}) \partial \mathrm{V} / \partial \mathrm{t}=0$

Both potentials satisfy the wave equation

$$
\begin{aligned}
& \nabla^{2} \mathrm{~V}-\left(1 / \mathrm{c}^{2}\right) \partial \mathrm{V} / \partial \mathrm{t}=0 \\
& \nabla^{2} \mathbf{A}-\left(1 / \mathrm{c}^{2}\right) \partial \mathbf{A} / \partial \mathrm{t}=0
\end{aligned}
$$

The symmetry of the geometry under consideration is such that V and A do not depend on the angular coordinate $\psi$. Moreover

$$
\begin{aligned}
& \mathrm{V}=\mathrm{V}(\eta, \theta) \cos (\omega \mathrm{t}) \\
& \mathbf{A}=\mathbf{A}(\eta, \theta) \sin (\omega \mathrm{t}) \\
& \mathbf{A}=\left[\mathrm{A}_{\eta}, \mathrm{A}_{\theta}, 0\right] \sin (\omega \mathrm{t})
\end{aligned}
$$

and

$$
\begin{aligned}
& \mathbf{E}=\left[\mathrm{E}_{\eta}, \mathrm{E}_{\theta}, \quad 0\right] \cos (\omega \mathrm{t}) \\
& \mathbf{B}=\left[0,0, \mathrm{~B}_{\psi}\right] \sin (\omega \mathrm{t})
\end{aligned}
$$

Using approximations similar to those of Appendix A valid in the region $\rho<$, we derive

$$
\begin{aligned}
& \mathrm{V}=\sum_{\mathrm{n}} \mathrm{a}_{\mathrm{n}} \mathrm{J}_{\mathrm{n}}(\mathrm{q} \rho) \sin (\mathrm{n} \theta) \cos (\omega \mathrm{t}) \\
& \mathrm{A}_{\eta}=-\sum_{\mathrm{n}} \mathrm{a}_{\mathrm{n}} \mathrm{J}_{\mathrm{n}+1}(\mathrm{q} \rho) \sin (\mathrm{n} \theta) \sin (\omega \mathrm{t}) \\
& \mathrm{A}_{\theta}=-\sum_{\mathrm{n}} \mathrm{a}_{\mathrm{n}} \mathrm{J}_{\mathrm{n}+1}(\mathrm{q} \rho) \cos (\mathrm{n} \theta) \sin (\omega \mathrm{t})
\end{aligned}
$$

Observe that because of the definition of the toroidal coordinate $\eta$, which increases in magnitude moving toward the center $\mathrm{P}$ of the CRFQ internal structure, the component $\mathrm{E}_{\eta}$ is positive when directed toward the center $\mathrm{P}$. At the same time, the component $\mathrm{E}_{\theta}$ is positive when directed clockwise. Transforming to the reduced toroidal coordinate system,

$$
\begin{aligned}
& E_{x}=E_{\eta} \cos \theta+E_{\theta} \sin \theta \\
& E_{z}=-E_{\eta} \sin \theta+E_{\theta} \cos \theta
\end{aligned}
$$


We retain only the lowest order term $\mathrm{n}=2$. Also, we shall assume that $\mathrm{qb}<<1$; that is, the RF wavelength $\lambda$ is considerably larger than the minor radius, $2 \pi b<<\lambda$. We obtain then, by using approximations similar to those of Appendix A valid in the region $\rho<b$ and by expanding the Bessel functions,

$$
\begin{aligned}
& E_{x}=2 V_{0}\left(z / b^{2}\right)\left[1+q^{2}\left(3 x^{2}-z^{2}\right) / 12\right] \cos (\omega t) \\
& E_{z}=2 V_{0}\left(x / b^{2}\right)\left[1+q^{2}\left(3 z^{2}-x^{2}\right) / 12\right] \cos (\omega t)
\end{aligned}
$$

which are derived from Eqs. (B1 and B2). The linear term is derived directly from the scalar potential $\mathrm{V}$. The higher order terms are the contribution from the vector potential A.

Similarly, the magnetic field component

$\mathrm{B}_{\psi}=\mathrm{V}_{0} \mathrm{q}\left[\left(\mathrm{x}^{2}-\mathrm{z}^{2}\right) / \mathrm{b}^{2}\right] \sin (\omega \mathrm{t})$

that being of higher order can be neglected. 


\section{Appendix C}

In terms of the local toroidal coordinates $(\rho, \theta, \psi)$, the convolution of the rods introduces a $\psi$-dependence in the wave equation for the scalar potential. That is, Eq. (1) is replaced by

$\nabla^{2} \mathrm{~V}(\rho, \theta, \psi)+\mathrm{q}^{2} \mathrm{~V}(\rho, \theta, \psi)=0$

Let us follow the same details of the computation shown in Appendix A, except noting that now a term $C \partial^{2} \mathrm{~V} / \partial \psi^{2}$ is to be added at the r.h. side of Eq. (A1), with

$\mathcal{C}(\eta, \theta)=1 / \sinh \eta(\cosh \eta-\cos \theta)$

In the region $\rho<b$, between the rods, and where $\eta$ is large, all the approximations of Appendix A are used again. Moreover,

$e \sim \beta \sim \rho^{2} / \mathrm{R}^{2}$

and Eq.(2) now becomes

$\rho^{2} \partial^{2} \mathrm{~V} / \partial \rho^{2}+\rho \partial \mathrm{V} / \partial \rho+\partial^{2} \mathrm{~V} / \partial \theta^{2}+\left(\rho^{2} / \mathrm{R}^{2}\right) \partial^{2} \mathrm{~V} / \partial \psi^{2}+\mathrm{q}^{2} \rho^{2} \mathrm{~V}=0$

The solution of this equation can be written as a double summation

$\mathrm{V}(\rho, \theta, \psi)=\Sigma_{\mathrm{n}, \mathrm{m}} \mathrm{a}_{\mathrm{nm}} \mathrm{f}_{\mathrm{nm}}(\rho) \sin (\mathrm{n} \theta) \cos (\mathrm{m} \psi) \cos (\omega \mathrm{t})$

By substitution in Eq. (C4) we derive the Bessel second-order differential equation

$$
\rho^{2} \partial^{2} f_{n m} / \partial \rho^{2}+\rho \partial f_{n m} / \partial \rho-\left(n^{2}+q_{m}^{2} \rho^{2}\right) f_{n m}=0
$$

which has for solution a Bessel function of either the first $J_{n}\left(\left|q_{m}\right| \rho\right)$ or the second kind $I_{n}\left(q_{m} \rho\right)$ depending on whether $q_{m}^{2}=m^{2} / R^{2}-q^{2}$ is negative or positive, respectively.

We shall assume that the origin $\psi=0$ corresponds to a minimum of the convolution as shown in Figure 7. Moreover, we retain in the double summation of Eq. (C5) only the lowest order terms. These are $\mathrm{n}=2$ and $\mathrm{m}=0$ corresponding to fields with no longitudinal dependence, equivalent to Eq. (4); and $\mathrm{n}=0$ and $\mathrm{m}=\mathrm{n}_{\mathrm{s}}$ where $\mathrm{n}_{\mathrm{s}}$ is the number of convolutions, that is, the number of periods per turn. Thus, with $\mathrm{q}_{\mathrm{m}}=\mathrm{k}_{\mathrm{s}} / \gamma_{\mathrm{s}}$,

$$
\begin{array}{rll}
\mathrm{V}(\rho, \theta, \psi) & = & \mathrm{a}_{20} \mathrm{~J}_{2}(\mathrm{q} \rho) \sin (2 \theta) \cos (\omega \mathrm{t}) \\
+ & \mathrm{a}_{0 \mathrm{~m}} \mathrm{I}_{0}\left(\mathrm{q}_{\mathrm{m}} \rho\right) \cos \left(\mathrm{n}_{\mathrm{s}} \psi\right) \cos (\omega \mathrm{t})
\end{array} \quad+
$$

The constants $\mathrm{a}_{20}$ and $\mathrm{a}_{0 \mathrm{~m}}$ are estimated by letting

$$
\mathrm{a}_{20} \mathrm{~J}_{2}\left(\mathrm{q} \mathrm{b}_{1}\right)+\mathrm{a}_{0 \mathrm{~m}} \mathrm{I}_{0}\left(\mathrm{q}_{\mathrm{m}} \mathrm{b}_{1}\right) \quad=\mathrm{V}_{0}
$$


$\mathrm{a}_{20} \mathrm{~J}_{2}\left(\mathrm{qb}_{2}\right)-\mathrm{a}_{0 \mathrm{~m}} \mathrm{I}_{0}\left(\mathrm{q}_{\mathrm{m}} \mathrm{b}_{2}\right) \quad=\mathrm{V}_{0}$

from which we derive

$\mathrm{a}_{20}=\mathrm{V}_{0}\left[\mathrm{I}_{0}\left(\mathrm{q}_{\mathrm{m}} \mathrm{b}_{2}\right)+\mathrm{I}_{0}\left(\mathrm{q}_{\mathrm{m}} \mathrm{b}_{1}\right)\right] /\left[\mathrm{J}_{2}\left(\mathrm{qb}_{1}\right) \mathrm{I}_{0}\left(\mathrm{q}_{\mathrm{m}} \mathrm{b}_{2}\right)+\mathrm{J}_{2}\left(\mathrm{qb}_{2}\right) \mathrm{I}_{0}\left(\mathrm{q}_{\mathrm{m}} \mathrm{b}_{1}\right)\right]$

$\mathrm{a}_{0 \mathrm{~m}}=\mathrm{V}_{0}\left[\mathrm{~J}_{2}\left(\mathrm{q} \mathrm{b}_{2}\right)-\mathrm{J}_{2}\left(\mathrm{q} \mathrm{b}_{1}\right)\right] /\left[\mathrm{J}_{2}\left(\mathrm{qb}_{1}\right) \mathrm{I}_{0}\left(\mathrm{q}_{\mathrm{m}} \mathrm{b}_{2}\right)+\mathrm{J}_{2}\left(\mathrm{qb} \mathrm{b}_{2}\right) \mathrm{I}_{0}\left(\mathrm{q}_{\mathrm{m}} \mathrm{b}_{1}\right)\right]$

In the case of smooth rods, with no convolution, $\mathrm{b}_{2}=\mathrm{b}_{1}=\mathrm{b}$ and $\mathrm{a}_{0 \mathrm{~m}}=0$ identically, whereas

$\mathrm{a}_{20}=\mathrm{V}_{0} / \mathrm{J}_{2}(\mathrm{qb})$

and thus one recovers the result given by Eq. (4).

More generally, the first term at the r.h. side of Eq. (C7) corresponds to the potential of Eq. (4) multiplied by a factor

$1-\xi=J_{2}\left(q b_{1}\right)\left[I_{0}\left(q_{m} b_{2}\right)+I_{0}\left(q_{m} b_{1}\right)\right] /\left[J_{2}\left(q b_{1}\right) I_{0}\left(q_{m} b_{2}\right)+J_{2}\left(q b_{2}\right) I_{0}\left(q_{m} b_{1}\right)\right]$

By treating the arguments of the Bessel functions as small compared to unit and by expanding we have

$$
\xi \quad=\quad\left[\left(b_{2} / b_{1}\right)^{2}-1\right] /\left[\left(b_{2} / b_{1}\right)^{2}+1\right]
$$

that, since $b_{2}>b_{1}$, has a value always between 0 and 1 .

Thus the transverse equations of motion Eqs. (15 and 16), or their coupled form Eq.s (23 and 24$)$, are still valid except that the restoring force is reduced by a factor $(1-\xi)$. As long as $b_{2} \approx b_{1}$ this reduction factor can be ignored.

The second term at the r.h. side of Eq. (C7) represents the contribution to the field of the rod convolution. In order to satisfy the Lorenz relation given by Eq. (B3) we need to add to this term also a vector potential that has only the $\eta$-component not vanishing. Following the approximations and the procedures of Appendices A and B we can write the new contribution to the potentials from the rod convolution as

$\mathrm{V}_{\mathrm{c}}=\mathrm{V}_{0} \xi\left[\mathrm{I}_{0}\left(\mathrm{q}_{\mathrm{m}} \rho\right) / \mathrm{I}_{0}\left(\mathrm{q}_{\mathrm{m}} \mathrm{b}_{1}\right)\right] \cos \left(\mathrm{n}_{\mathrm{s}} \psi\right) \cos (\omega \mathrm{t})$

$\mathrm{A}_{\mathrm{c}}=-\mathrm{V}_{0} \xi\left[\mathrm{I}_{1}\left(\mathrm{q}_{\mathrm{m}} \rho\right) / \mathrm{I}_{0}\left(\mathrm{q}_{\mathrm{m}} \mathrm{b}_{1}\right)\right] \cos \left(\mathrm{n}_{\mathrm{s}} \psi\right) \sin (\omega \mathrm{t})$

where $\xi$ is given in good approximation by Eq. (C14).

The potentials given by Eqs. (C15 and C16) give rise to an extra transverse electric field which is linear in the displacements $\mathrm{x}$ and $\mathrm{z}$. This field should be added to the equations of motion Eqs. (15 and 16), Nevertheless, as long as $b<<\mathrm{L}_{\mathrm{s}}$ this field is small and could in principle be ignored. The effect of this field will not be considered further. 
The potentials combined of Eqs. (C15 and C16) give rise to a longitudinal electric field, that, on the axis $\rho=0$, is

$$
\begin{aligned}
\mathrm{E}_{\psi} & =-(1 / \mathrm{R}) \partial \mathrm{V}_{\mathrm{c}} / \partial \psi \\
& =\mathrm{V}_{0} \xi \mathrm{k}_{\mathrm{s}} \sin \left(\mathrm{n}_{\mathrm{s}} \psi\right) \cos (\omega \mathrm{t})
\end{aligned}
$$




\section{Appendix D}

The general equations of motion are given by Eq.s $(54,55)$. We shall describe the solution of Eq. (54). That of Eq. (55) is then obtained by shifting $\mathrm{k}_{\mathrm{s}}$ of 180 degrees. It is convenient to replace the path length $\mathrm{s}$ with the angular variable

$\theta \quad=\quad \pi \mathrm{s} / \mathrm{L}$

and the displacement $u$ with the scaled variable

$\mathrm{w}(\theta)=\left(\pi^{2} \underline{\mathrm{R}} / \mathrm{L}^{2}\right) \mathrm{u}(\mathrm{s})$

so that Eq. (54) reduces to

$\mathrm{d}^{2} \mathrm{w} / \mathrm{d} \theta^{2}-2 \mathrm{qw} \cos (2 \theta)-\Delta \mathrm{w}=1$

where

$\mathrm{q}=\mathrm{B} / 2 \pi^{2}$

$\Delta \quad=\quad \mathrm{KL}^{2} / \pi^{2} \mathrm{a}^{2}$

and $\mathrm{K}$ is the space-charge parameter defined by Eq. (56). The total solution of Eq. (D3) is given by the sum of the general solution of the homogenous equation

$\mathrm{d}^{2} \mathrm{w} / \mathrm{d} \theta^{2}-2 \mathrm{qw} \cos (2 \theta)-\Delta \mathrm{w}=0$

that can be expressed in terms of the Mathieu functions of the first kind $\operatorname{Ce}(-\Delta, \mathrm{q}, \theta)$ and $\operatorname{Se}(-\Delta, \mathrm{q}, \theta)$, and of a particular solution $\underline{\mathrm{w}}(\theta)$, periodic in $\theta$ with period $\pi$, of the inhomogenous Eq. (D3) itself, namely

$\mathrm{w}(\theta)=\mathrm{c}_{1} \operatorname{Ce}(-\Delta, \mathrm{q}, \theta)+\mathrm{c}_{2} \operatorname{Se}(-\Delta, \mathrm{q}, \theta)+\underline{\mathrm{w}}(\theta)$

where $c_{1}$ and $c_{2}$ are two constants to be determined from initial conditions. The motion described by Eq. (D7) is that of a free betatron oscillation around a closed orbit of period $\mathrm{L}$ in the path length $\mathrm{s}$, and of period $\pi$ in the arc length $\theta$. The free betatron oscillation has a betatron frequency that can be expressed by the betatron tune per period $v$, that is the phase advance per period normalized to $2 \pi$. It is plotted in Figure 17 versus the RFQ parameter B given by Eq. (18). The corresponding amplitude $\beta_{\mathrm{L}}$-function, equivalent to that of a FODO focusing structure, is plotted in Figures 18 and 19. 


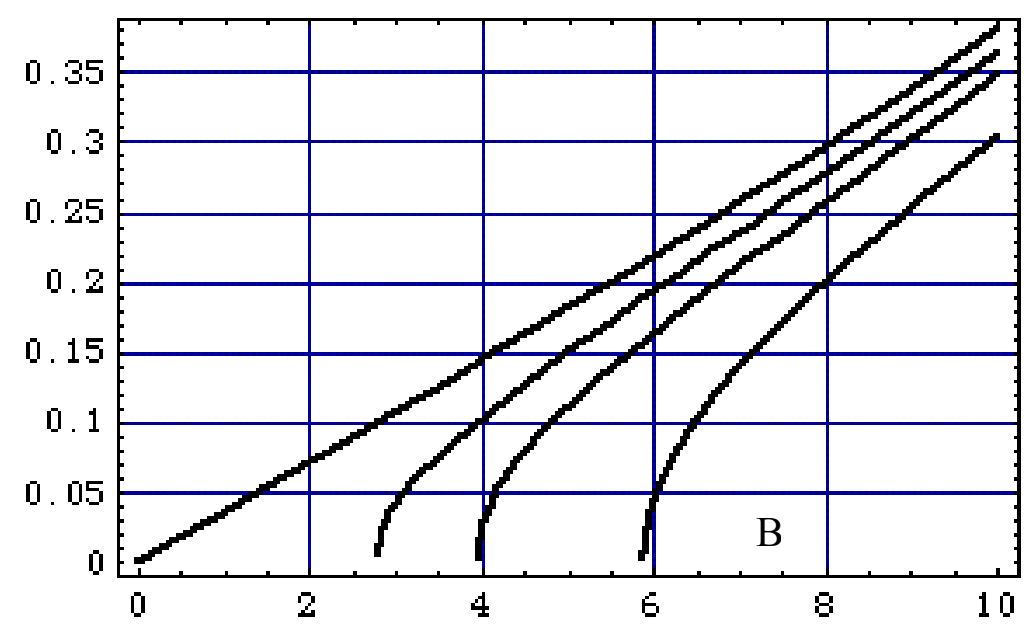

Figure 17. Betatron Tune $v$ per period versus the RFQ parameter B. The top curve corresponds to $\Delta=0$, and the others, in descending order, to $\Delta=0.01,0.02$, and 0.044 .

The rotation of the beam ellipse in the phase space is given by the Twiss parameter $\alpha_{L}=-(1 / 2) d \beta_{L} / d s$. The maximum absolute value of this parameter is plotted in Figure 20.

Inspection of Figures 17, 19 and 20 clearly indicates a region of motion stability that is allowed by the presence of space charge forces. The larger is the space charge parameter $\Delta$ the narrower is the stability region.

The closed orbit distortion is a solution of Eq. (D7), periodic with $\theta$ with period $\pi$. For its determination the two constants $\mathrm{c}_{1}$ and $\mathrm{c}_{2}$ appearing in Eq. (D7) are solved for so that $\mathrm{w}(\pi)=\mathrm{w}(0)$ and $\mathrm{w}^{\prime}(\pi)=\mathrm{w}^{\prime}(0)$. We remind that $\mathrm{Se}(0)=\mathrm{Ce}^{\prime}(0)=0$. We also impose that $\underline{\mathrm{w}}(0)=\underline{\mathrm{w}}^{\prime}(0)=0$ by choosing the particular solution of the in-homogeneous Eq. (D3) in the form

$\underline{\mathrm{w}}(\theta)=\int\left\{[\operatorname{Se}(\theta) \operatorname{Ce}(\mathrm{t})-\operatorname{Ce}(\theta) \operatorname{Se}(\mathrm{t})] /\left[\operatorname{Ce}(\mathrm{t}) \operatorname{Se}^{\prime}(\mathrm{t})-\operatorname{Se}(\mathrm{t}) \operatorname{Ce}^{\prime}(\mathrm{t})\right]\right\} d \mathrm{~d}$

where the integral is between 0 and $\theta$. The resulting orbit distortion $\mathrm{w}_{\mathrm{c}}(\theta)$ is plotted in Figures 21 and 22. 


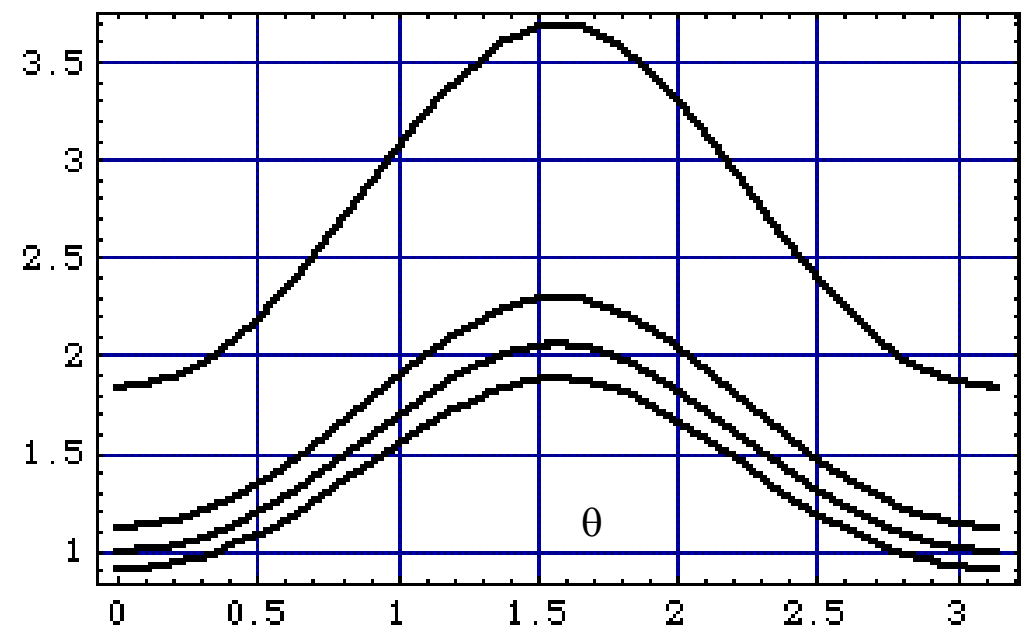

Figure 18. The ratio $\beta_{\mathrm{L}} / \mathrm{L}$ over the length of a Period $\mathrm{L}$ normalized between 0 and $\pi$, for $\mathrm{B}=6.80928$. The top curve corresponds to $\Delta=0.044$, and the others, in descending order, to $\Delta=0.02,0.01$, and 0.0 .

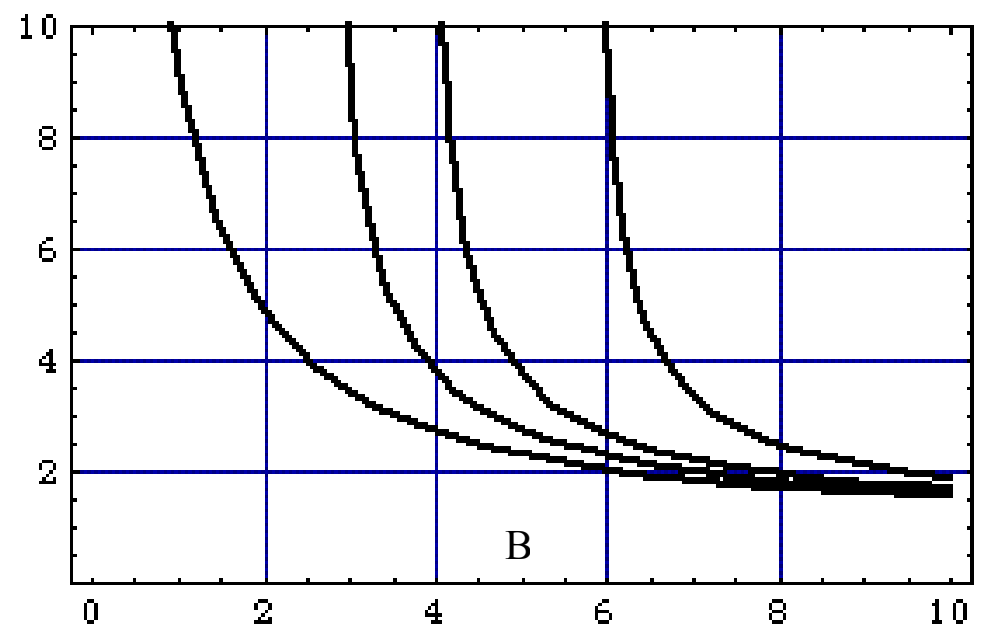

Figure 19. The maximum value of the ratio $\beta_{\mathrm{L}} / \mathrm{L}$ versus the RFQ parameter $\mathrm{B}$. The curve to the left corresponds to $\Delta=0$, and the others, moving toward the right, to $\Delta=0.01,0.02$, and 0.044 . 


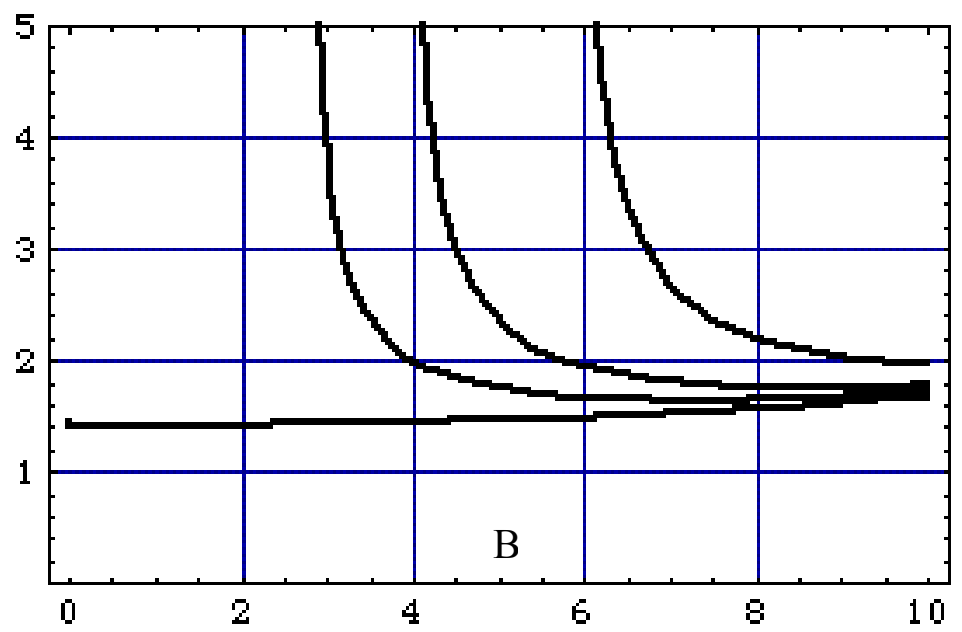

Figure 20. The maximum absolute value of $\alpha_{L}$ versus the RFQ parameter $B$. The curve to the bottom corresponds to $\Delta=0$, and the others, moving toward the right, to $\Delta=0.01$, 0.02 , and 0.044 .

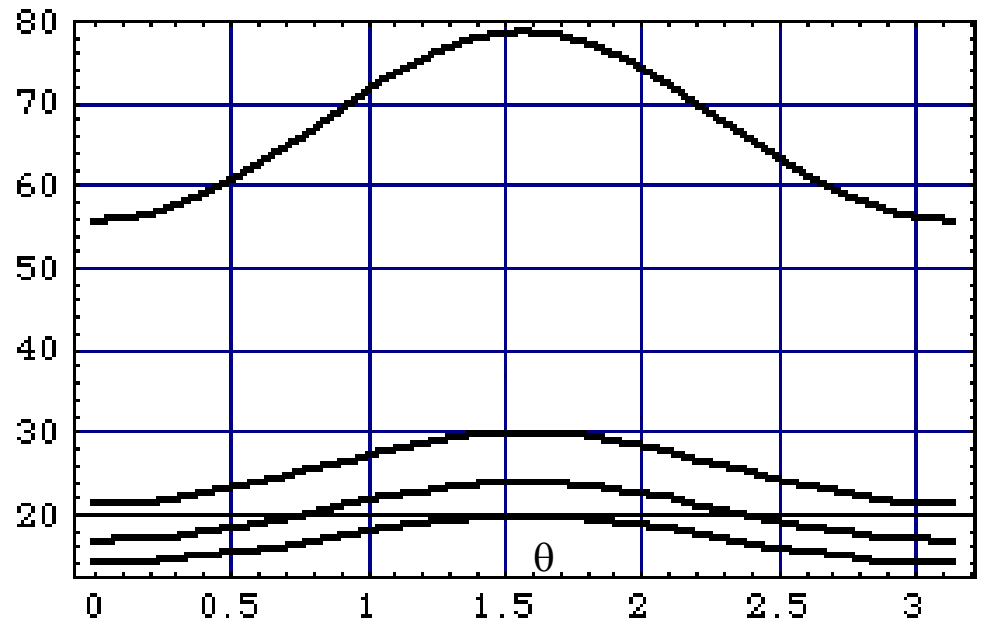

Figure 21. The orbit distortion $\mathrm{w}_{\mathrm{c}}(\theta)$ over the length of a Period $\mathrm{L}$ normalized between 0 and $\pi$, for $\mathrm{B}=6.80928$. The top curve corresponds to $\Delta=0.044$, and the others, in descending order, to $\Delta=0.02,0.01$, and 0.0 .

The actual orbit distortion $\mathrm{u}_{\mathrm{c}}(\mathrm{s})$ is related to the reduced orbit distortion $\mathrm{w}_{\mathrm{c}}(\theta)$ through the relation

$\mathrm{u}_{\mathrm{c}}(\mathrm{s})=\mathrm{w}_{\mathrm{c}}(\theta) \mathrm{L}^{2} / \pi^{2} \underline{\mathrm{R}}$

where $\mathrm{w}_{\mathrm{c}}(\theta)$ acts as a weighting universal function. In the special case that $1 / \underline{R}=0$ as, for instance in the case where the bending radius $r_{L}$ of the external magnetic field cancels exactly the geometrical radius $R$, the actual physical orbit distortion $\mathrm{u}_{\mathrm{c}}(\mathrm{s})$ vanishes identically no matter what is the value of the $\mathrm{w}_{\mathrm{c}}(\theta)$ function. 
The path length per period $\mathrm{L}$ is increased by the physical orbit distortion. The relative increase can be determined as follows

$$
\Delta \mathrm{L} / \mathrm{L}=\left(\mathrm{L}^{2} / \pi^{3} \mathrm{R} \underline{\mathrm{R}}\right) \int_{\mathrm{W}_{\mathrm{c}}}(\theta) \mathrm{d} \theta
$$

where the integral extends over the arc of a period, from 0 to $\pi$. Again, in the limit $1 / \underline{R}=$ 0 the increase of the path length vanishes identically. The integral $I=\int_{W_{c}}(\theta) d \theta$ is plotted in Figure 23.

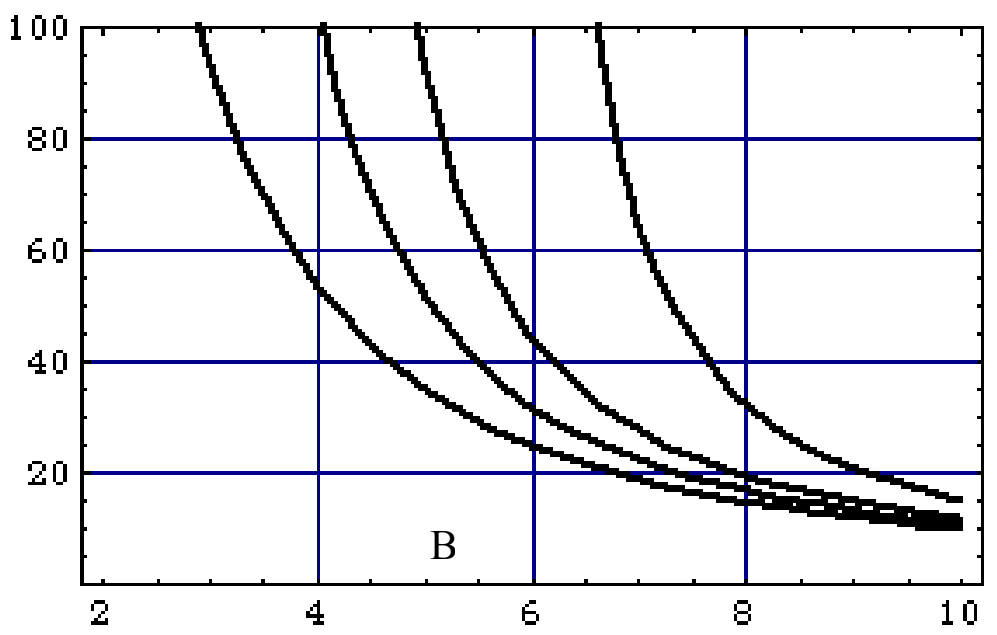

Figure 22. The maximum value of the orbit distortion $\mathrm{w}_{\mathrm{c}}(\theta)$ versus the RFQ parameter $\mathrm{B}$. The curve to the left corresponds to $\Delta=0$, and the others, moving toward the right, to $\Delta=0.01,0.02$, and 0.044 .

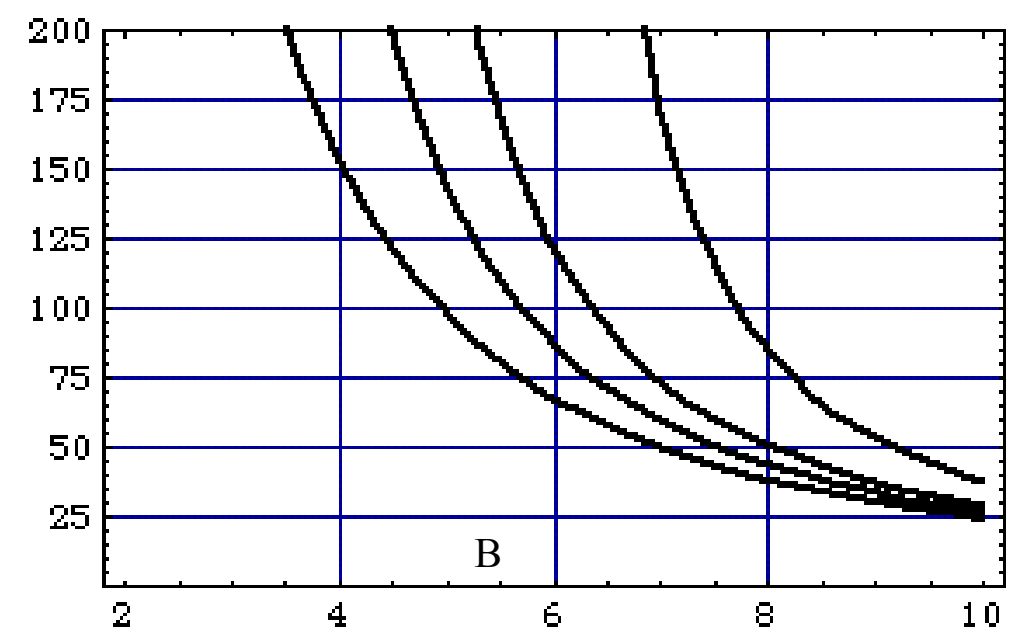

Figure 23. The plot of the integral $I=\int_{W_{c}}(\theta) d \theta$ needed to determine the relative path length increase due to the orbit distortion (Eq.(D10)), versus the RFQ parameter B. The curve to the left corresponds to $\Delta=0$, and the others, moving toward the right, to $\Delta=0.01,0.02$, and 0.044 . 
The dispersion function $\eta(s)$ is the displacement from the physical orbit distortion $u_{c}(s)$ due a momentum error $\delta$ of the particle. In linear approximation we can write

$$
\mathrm{u}_{\mathrm{c}}(\mathrm{s})=\mathrm{u}_{\mathrm{co}}(\mathrm{s})+\eta(\mathrm{s}) \delta
$$

where $u_{\mathrm{co}}(\mathrm{s})$ is the central value of the orbit distortion, given by Eq. (D9) with $\delta=0$. We shall consider two cases. In the first case there is an external bending magnet with a field to cancel exactly the geometrical radius of curvature, that is $r_{L s}=R$. In this case the dispersion is exactly equal to the orbit distortion itself (with $\underline{\mathrm{R}}$ replaced by $\mathrm{R}$ )

$\eta(\mathrm{s})=\mathrm{u}_{\mathrm{co}}(\mathrm{s})$

In the other extreme case where there is no external bending field, $\underline{\mathrm{R}}=\mathrm{R}$, and

$\eta(\mathrm{s})=\mathrm{u}_{\mathrm{co}}(\mathrm{s})\left(2 / \gamma_{\mathrm{s}}^{2}-\chi_{\mathrm{w}} \beta_{\mathrm{s}}^{2}\right)$

where the maximum value of $\chi_{w}=\left(B / w_{c}\right)\left(\partial w_{c} / \partial B\right)$ is plotted in Figure 24 . Thus the dispersion follows closely the behavior of the orbit distortion.

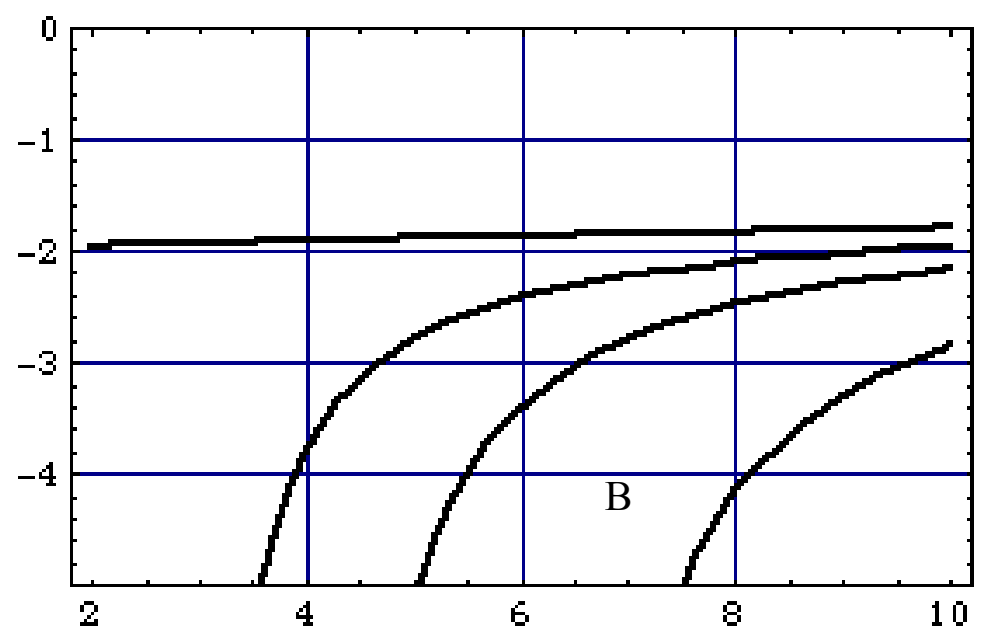

Figure 24. Plot of the maximum value of $\chi_{w}=\left(B / w_{c}\right)\left(\partial w_{c} / \partial B\right)($ at $\theta=\pi / 2)$ versus $B$. The top curve corresponds to $\Delta=0$, and the others, in descending, order to $\Delta=0.01$, 0.02 , and 0.044 .

The momentum compaction factor $\alpha_{\mathrm{p}}$, that is the relative change $\Delta \mathrm{L} / \mathrm{L}$ of the path length per unit of the relative change $\delta$ of the momentum error is essentially given by Eq. (D10). More precisely

$$
\alpha_{\mathrm{p}} \quad=(\mathrm{L} / \pi \mathrm{R})^{2} \mathrm{If} / \pi
$$


where $I=\int_{W_{c}}(\theta) d \theta$ is plotted in Figure 23; $f=1$ with an external bending field such that $r_{L s}=R$, and $f=2 / \gamma_{s}{ }^{2}-\chi_{w} \beta_{s}{ }^{2}$ in the case there is no external bending magnet. From Eq. (D14) we can also derive the transition energy

$$
\gamma_{\mathrm{T}}=\left(1 / \alpha_{\mathrm{p}}\right)^{1 / 2}=(\pi \mathrm{R} / \mathrm{L})(\pi / \mathrm{If})^{1 / 2}
$$

The quantity $(\pi / \mathrm{I})^{1 / 2}$ is plotted in Figure 25 .

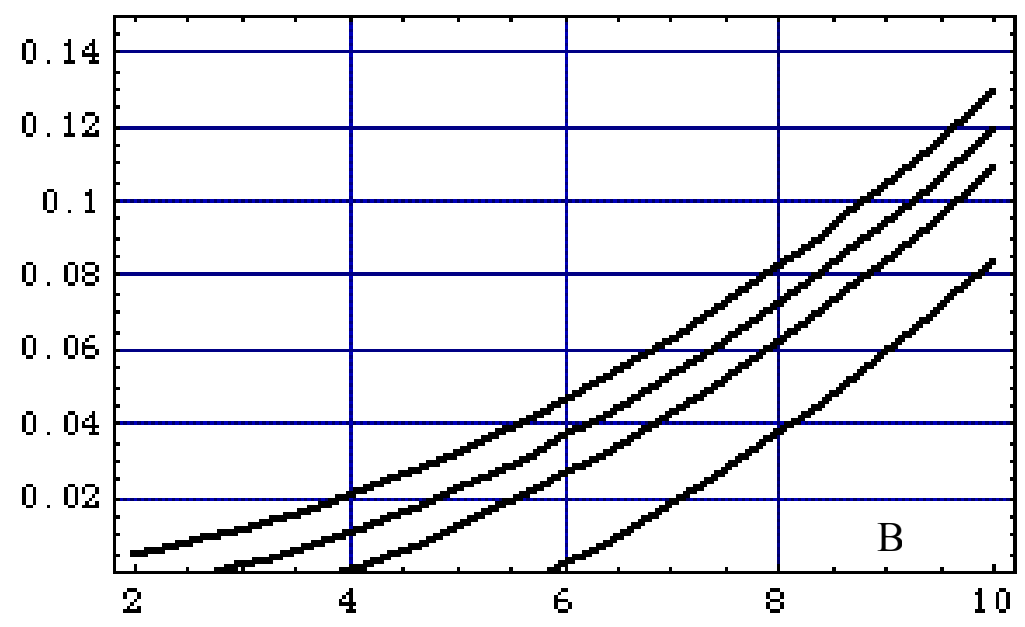

Figure 25. Plot of $(\pi / \mathrm{I})^{1 / 2}$, that is related to the transition energy $\gamma_{\mathrm{T}}$, versus the RfQ parameter B. The top curve corresponds to $\Delta=0$, and the others, in descending, order to

$$
\Delta=0.01,0.02 \text {, and } 0.044 \text {. }
$$

The number $v$ of betatron oscillations per period is related to the phase advance $\mu=2 \pi v$ per period, and that depends only on the RFQ parameter B. The natural chromaticity is the relative variation of the betatron tune $v$ with the relative momentum error $\delta$

$$
\xi_{\mu} \quad=\quad(1 / v) \mathrm{d} v / \mathrm{d} \delta=\quad-\beta_{\mathrm{s}}^{2} \chi_{\mu}
$$

where $\chi_{\mu}=(B / v) \partial v / \partial B$ is plotted in Figure 26. Similarly, the amplitude lattice function $\beta_{\mathrm{L}}$ varies with the momentum error $\delta$, which is another chromatic effect. The ratio $\beta_{\mathrm{L}} / \mathrm{L}$ depends only on the RFQ parameter B and we can define

$$
\xi_{\beta}=\left(\mathrm{L} / \beta_{\mathrm{L}}\right) \mathrm{d}\left(\beta_{\mathrm{L}} / \mathrm{L}\right) / \mathrm{d} \delta=-\beta_{\mathrm{s}}^{2} \chi_{\beta}
$$

where $\chi_{\beta}=\left(\mathrm{BL} / \beta_{\mathrm{L}}\right) \partial\left(\beta_{\mathrm{L}} / \mathrm{L}\right) / \partial \mathrm{B}$ is plotted in Figure 27 . To get the actual variation of $\beta_{\mathrm{L}}$ alone with the momentum error $\delta$, one should multiply the ratio $\beta_{\mathrm{L}} / \mathrm{L}$ by the period $\mathrm{L}$ that has its own momentum dependence as $\mathrm{L}=\mathrm{L}_{\mathrm{s}}\left(1+\delta / \gamma_{\mathrm{s}}{ }^{2}\right)$.

We have seen that the dispersion $\eta$ is the variation of the orbit distortion with the momentum error. But, to second order, also the dispersion has a chromatic effect and it varies with the momentum error $\delta$. We shall not calculate this here. 


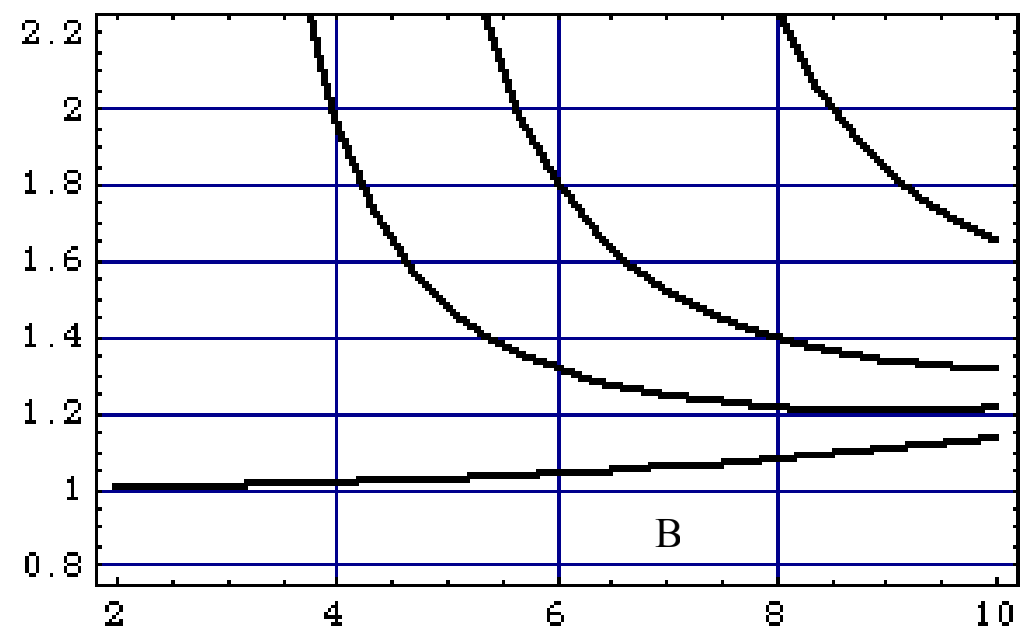

Figure 26. The tune chromaticity $\chi_{\mu}=(\mathrm{B} / v) \partial v / \partial \mathrm{B}$ versus the RFQ parameter $\mathrm{B}$. The bottom curve corresponds to $\Delta=0$, and the others, in ascending, order to $\Delta=0.01,0.02$, and 0.044 .

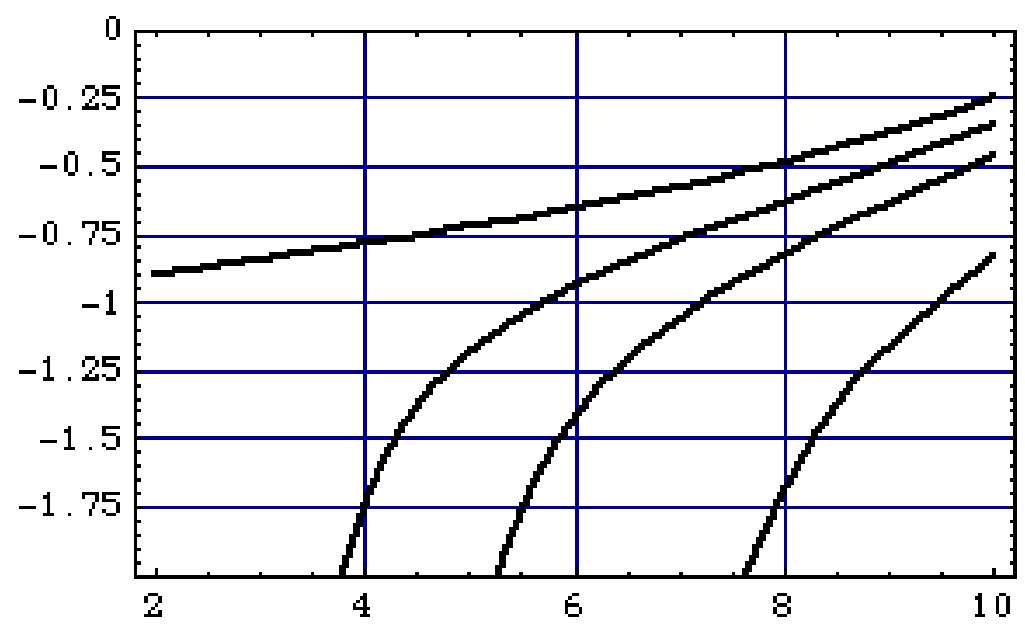

Figure 27. The amplitude chromaticity $\chi_{\beta}=\left(\mathrm{BL} / \beta_{\mathrm{L}}\right) \partial\left(\beta_{\mathrm{L}} / \mathrm{L}\right) / \partial \mathrm{B}$, estimated in the middle of a period where has the maximum value, versus the RFQ parameter $B$. The top curve corresponds to $\Delta=0$, and the others, in descending, order to $\Delta=0.01,0.02$, and 0.044 . 